\title{
Modeling and simulation of liquid pulsed particulate fluidized beds
}

\author{
Guodong Jin, Dayou Liu* \\ DES, Institute of Mechanics, Chinese Academy of Sciences, Beijing, 100080, PR China \\ MPR, Institute of Process Engineering, Chinese Academy of Sciences, Beijing, 100080, PR China
}

Received 13 November 2004; received in revised form 29 March 2005; accepted 2 May 2005

Available online 14 June 2005

\begin{abstract}
Pulsed fluidization is of considerable interest in process engineering for improving fluidization quality. Quantitative understanding of the pulsed two-phase flow behaviors is very important for proper design and optimum operation of such contactors. The main objective of this study is to understand the mathematical models for liquid pulsed particulate fluidization and its dynamic processes. The state of the arts of the Two-Fluid Model (TFM) and its constitutive relationships are discussed and then, the Local Equilibrium Model (LEM), a simplified version of TFM, is developed and solved for liquid pulsed fluidization. LEM is proposed to describe the liquid pulsed fluidization with acceptable engineering accuracy as compared with the experimental data, and its shortcomings are also discussed at length by analyzing the relaxation processes of the two-phase flow due to a jump change of fluidizing velocity and the structure of concentration discontinuity which forms in the bed collapse process. The main shortcoming of LEM is that some detailed information in the flow fields is lost. However, errors only exist in a time interval of tens of milliseconds (several times of particle relaxation time) and a spatial span of several millimeters (several times of particle diameter).
\end{abstract}

(C) 2005 Elsevier B.V. All rights reserved.

Keywords: Fluidization; Pulsed flow; Two-Fluid Model; Local equilibrium model; Numerical simulation; Concentration wave

\section{Introduction}

Fluidized beds are common and important reactors in process engineering because of the good mass and heat transfer rate between the fluid and the particles, and between the particles and the sidewall. It is well known that there are non-uniform flow structures such as bubbles and slugs in fluidization [1]. Large bubbles and slugs are undesirable for efficient operations, because they can reduce the contact efficiency.

Many studies show that fluidization quality can be much improved by externally applied vibrations or pulsations [28]. As a method of eliminating slugs and gas channeling, reducing the size of bubbles, thus improving the fluidization quality, pulsed fluidization is an operation in which the fluidizing velocity $U(t)$ pulsates with time as a

\footnotetext{
* Corresponding author. Tel.: +86 10 62573765; fax: +86 1062561284 .

E-mail address: Dyliu@imech.ac.cn (D. Liu)
}

rectangular wave, a cosinoidal wave or any other patterns. Pulsed-stabilized fluidization can increase the heat transfer coefficient when used for coal combustion [7]. Pulsation can suppress the bubble flow, produce more uniform fluidization and increase the gas-solid contact efficiency [5]. Although many people have studied the behaviors of the transient flows in fluidized beds [9-17], however, the flow patterns of unsteady two-phase flows are very complex, the quantitative understanding of the flow in pulsed fluidized beds is far from being complete. Earlier papers have usually focused on predicting the bed height variation in the simple processes such as the bed expansion or bed collapsing (bed contraction) when the fluidizing velocity suddenly changes, corresponding to very low pulsation frequency of $U(t)$. Few papers predicted the bed height, especially the distribution of particle concentration along the bed height when the pulsation frequency of $U(t)$ is not very low.

In order to quantitatively understand the complex flow behaviors in the pulsed fluidized beds, as the first step, the 
main aim of this study is to select and develop proper mathematical models for liquid pulsed particulate fluidization, predict the dynamic processes in the beds and provide a basis for understanding more complex gas pulsed fluidization. The direct application of the particulate fluidization is encountered in most liquid fluidized beds and some gas fluidized beds, for example, $\mathrm{SiO}_{2}$ nano-scale particles are recently found to be particulately fluidized with porous multi-stage agglomerates [18].

In despite of some phenomenological analogies between liquid particulate fluidization and gas-solid particulate fluidization, there exist certain differences in mechanisms of inter-particle interactions and dynamic responses of phases. The most noteworthy ones includes (1) the inertia difference between the two-phase flows (same order in solidliquid fluidization but three order-of-magnitude in gas-solid fluidization) and the resulted dynamic responses in pulsed flows, (2) the inter-particle collisions (very weak interparticle collision in liquid fluidization versus extremely strong collision in gas fluidization which could not be ignored, especially for dense gas-solid flow), (3) electrostatic charge effects (very weak in liquid but strong in gasfine solid case), and (4) wall boundary effects (impedance due to fluid-solid wall interaction). Because of the above mentioned differences in liquid fluidization and gas fluidization, the developed model and the conclusions are restricted to the area of liquid pulsed fluidization and one should be careful to extend the liquid-solid modeling directly to gas-solid fluidization without capturing all the major mechanism differences.

The Two-Fluid Model (TFM) is commonly used to describe the fluid-particle two-phase flow, with the fluid and particles assumed as interpenetrating phases and coupled with mass, momentum or energy exchange [1922]. The key point is that the equation set must be closed with appropriate constitutive relationships, which makes the model rather complex. Although many constitutive relations are still being studied up to now, the Two-Fluid Model (or Multi-Fluid Model) has been widely used for modeling, analyzing and simulating of fluidization systems, and has become a useful tool for design and scale up of efficient reactors $[23,24]$.

In our liquid pulsed fluidization experiment, the onedimensional characteristic of the flow, i.e., the distinct planar wave characteristic of particle concentration in the bed can be obviously seen. The concentration waves (expansion wave and shock wave) travel upwards from the distributor $(x=0)$ (See Fig. 1). Fig. 1 shows the transient distribution of particle concentration in the entire column of the test section along the bed height at different times in a period, recorded by a digital camera. The test section is the whole bed just from above the distributor $(x=0.04 \mathrm{~m})$ to the bed surface $h(t)$. The gray scale of the photos represents the particle concentration: the darker the photo, the denser the particle concentration and the accurate digital results of solid fraction in Fig. 1 are shown in Fig. 11(a-f). The experimental systems, measurement method and measurement uncertainty analysis are described in Section 4. From Fig. 1, one can know that the bed height $h(t)$ oscillates up and down in a cycle, a concentration wave from the distributor $(x=0)$ periodically travels upward as a result of the periodical change of the fluidizing velocity $U(t)$, the dilute and dense sections of particle concentration distribute alternately, accompanying concentration discontinuities $a, b$ and $c$ between the dilute and dense sections. Meanwhile, the particle concentration along the bed height distributes quite uniformly at any cross-section, and wall boundary effect is inconspicuous. The above facts show that the axial movement of the two-phase flow prevails in the pulsed fluidized bed. As a first approximation, it is appropriate to use the onedimensional mathematical model to simulate the main characteristic of the flow, i.e., particles moving up and down.

In a steady state of a conventional fluidization, the crosssectional averaged particle velocity is zero, and the nonuniformity of the axial velocity at different radial points in a cross-section (such as core-annulus flow) is obvious when compared with the zero cross-sectional averaged velocity, the radial velocity also becomes important, thus the multidimensional flow structures exists in a statistically steadily fluidized bed $[25,26]$. In the pulsed fluidization, although

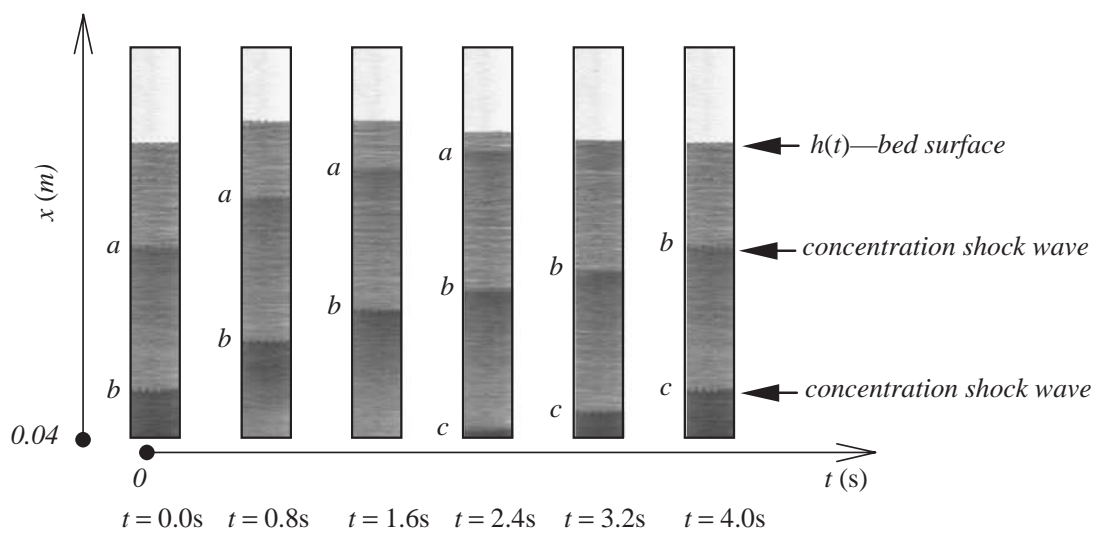

Fig. 1. Transient distribution of particle concentration in a pulsed fluidized bed when $U(t)$ varies with time as a rectangular wave with a period of $4 \mathrm{~s}$ (semi-on period $1 \mathrm{~s}$ and semi-off period $3 \mathrm{~s}$ ), the numerical value of bed height and solid volume fraction can be seen in Figs. 10 and $11(\mathrm{a}-\mathrm{f})$ respectively. 
the axial velocity is also non-uniform at different radial points and the radial velocity is not always small, however, their importance becomes secondary when compared with the large cross-sectional averaged axial velocity. Multidimensional models can indeed provide much more detailed information in the bed, however, one's attention may be scattered by these details and some important flow characteristics might be ignored (for example, the wave characteristic of the particle concentration in pulsed fluidization). If one mainly concerns the non-uniform flow profile or the radial mass and momentum transfer, a multidimensional model is necessary [27]; however, if one mainly concerns the variation of flow parameters with time and along the bed height, a one-dimensional model still has its advantage: the model is simple and helps to capture the main physical characteristic of the flow. Up to now, the unsteady flow in pulsed fluidized beds is not fully understood, the results gotten from the one-dimensional model is still of importance from both a theoretical and practical point of view. It is shown that the numerical results of the one-dimensional model well fit the experimental data as a whole.

The fluidizing velocity $U(t)$ is limited in the region between the minimum fluidizing velocity $U_{\mathrm{mf}}$ and the particle settling velocity $U_{\mathrm{T}},\left(U_{\mathrm{mf}}<U(t)<U_{\mathrm{T}}\right)$, which excludes the persistent contacting between particles and the case of particle free in a region near the distributor. This limitation guarantees the continuum assumption for solid phase valid and the application of Richardson-Zaki and Ergun equation appropriate.

\section{Two-Fluid Model}

The model used in this study is based on the following assumptions: the two phases are incompressible, the fluid density $\rho_{\mathrm{f}}$ and the particle density $\rho_{\mathrm{p}}$ are constants; the ratio of the bed diameter and the particle diameter is large enough to ignore the drag force of the sidewall; the gradient of viscous normal stress of fluid phase $\tau_{\mathrm{f}, \mathrm{xx}}$ is ignored because it is much smaller than that of fluid pressure. Under the above assumptions, the equations used by Anderson et al. [28] and Duru et al. [29] can be written as

$$
\begin{aligned}
& \frac{\partial \alpha_{\mathrm{p}}}{\partial t}+\frac{\partial\left(\alpha_{\mathrm{p}} u_{\mathrm{p}}\right)}{\partial x}=0, \\
& \frac{\partial \alpha_{\mathrm{f}}}{\partial t}+\frac{\partial\left(\alpha_{\mathrm{f}} u_{\mathrm{f}}\right)}{\partial x}=0 \\
& \rho_{\mathrm{p}}\left[\frac{\partial\left(\alpha_{\mathrm{p}} u_{\mathrm{p}}\right)}{\partial t}+\frac{\partial\left(\alpha_{\mathrm{p}} u_{\mathrm{p}}^{2}\right)}{\partial x}\right] \\
& =-\alpha_{\mathrm{p}} \frac{\partial p}{\partial x}+\frac{\partial\left(-p_{\mathrm{p}}+\tau_{\mathrm{p}, \mathrm{xx}}\right)}{\partial x}-\alpha_{\mathrm{p}} \rho_{\mathrm{p}} g+F_{\mathrm{p}},
\end{aligned}
$$

$\rho_{\mathrm{f}}\left[\frac{\partial\left(\alpha_{\mathrm{f}} u_{\mathrm{f}}\right)}{\partial t}+\frac{\partial\left(\alpha_{\mathrm{f}} u_{\mathrm{f}}^{2}\right)}{\partial x}\right]=-\alpha_{\mathrm{f}} \frac{\partial p}{\partial x}-\alpha_{\mathrm{f}} \rho_{\mathrm{f}} g-F_{\mathrm{p}}$

$\alpha_{\mathrm{p}}+\alpha_{\mathrm{f}}=1$.

where $\alpha_{\mathrm{p}}, \alpha_{\mathrm{f}}, u_{\mathrm{p}}$, and $u_{\mathrm{f}}$ are the volume fraction and velocity of the particle phase and fluid phase respectively, $F_{\mathrm{p}}$ is the inter-phase force per unit volume except for buoyancy ${ }^{1}, p$ is the fluid phase pressure, $p_{\mathrm{p}}, \tau_{\mathrm{p}, \mathrm{xx}}$ are particle phase pressure and viscous stress respectively.

Adding Eq. (1) to Eq. (2) and integrating the result along the bed height, one can get the continuity equation for the liquid-solid mixture to substitute Eq. (2)

$\alpha_{\mathrm{p}} u_{\mathrm{p}}+\alpha_{\mathrm{f}} u_{\mathrm{f}}=U(t)$.

Eliminating $\partial p / \partial x$ and $F_{\mathrm{p}}$ from Eqs. (3) and (4), respectively, one can obtain the equation concerning the inertial difference between the two phases and the liquidsolid mixture momentum equation

$$
\begin{gathered}
\rho_{\mathrm{p}}\left(\frac{\partial u_{\mathrm{p}}}{\partial t}+u_{\mathrm{p}} \frac{\partial u_{\mathrm{p}}}{\partial x}\right)-\rho_{\mathrm{f}}\left(\frac{\partial u_{\mathrm{f}}}{\partial t}+u_{\mathrm{f}} \frac{\partial u_{\mathrm{f}}}{\partial x}\right) \\
=\frac{F_{\mathrm{p}}}{\alpha_{\mathrm{p}} \alpha_{\mathrm{f}}}-\left(\rho_{\mathrm{p}}-\rho_{\mathrm{f}}\right) g+\frac{1}{\alpha_{\mathrm{p}}} \frac{\partial\left(-p_{\mathrm{p}}+\tau_{\mathrm{p}, \mathrm{xx}}\right)}{\partial x}, \\
\frac{\partial}{\partial t}\left(\alpha_{\mathrm{p}} \rho_{\mathrm{p}} u_{\mathrm{p}}+\alpha_{\mathrm{f}} \rho_{\mathrm{f}} u_{\mathrm{f}}\right)+\frac{\partial}{\partial x}\left(\alpha_{\mathrm{p}} \rho_{\mathrm{p}} u_{\mathrm{p}}^{2}+\alpha_{\mathrm{f}} \rho_{\mathrm{f}} u_{\mathrm{f}}^{2}\right) \\
=-\frac{\partial p}{\partial x}+\frac{\partial\left(-p_{\mathrm{p}}+\tau_{\mathrm{p}, \mathrm{xx}}\right)}{\partial x}-\left(\alpha_{\mathrm{p}} \rho_{\mathrm{p}}+\alpha_{\mathrm{f}} \rho_{\mathrm{f}}\right) g .
\end{gathered}
$$

The five unknowns $\alpha_{\mathrm{p}}, \alpha_{\mathrm{f}}, u_{\mathrm{p}}, u_{\mathrm{f}}$ and $p$ can be calculated by solving the equation set Eqs. (1)-(5). Substituting Eqs. $\left(3^{\prime}\right)$ and $\left(4^{\prime}\right)$ for Eqs. (3) and (4), one can solve the equation set (1), (2'), (3') and (5) to obtain $\alpha_{\mathrm{p}}, \alpha_{\mathrm{f}}, u_{\mathrm{p}}, u_{\mathrm{f}}$, then obtain $p$ by integrating Eq. ( $\left.4^{\prime}\right)$ along the bed height.

$F_{\mathrm{p}}$ includes the inter-phase drag force $F_{\mathrm{D}}$, the virtual mass force $F_{\mathrm{vm}}$ and so on,

$F_{\mathrm{p}}=F_{\mathrm{D}}+F_{\mathrm{vm}}+\cdots \cdots \cdot$

Introducing the momentum exchange coefficient $\beta$, one can express the drag force $F_{\mathrm{D}}$ as

$F_{\mathrm{D}}=\beta\left(u_{\mathrm{f}}-u_{\mathrm{p}}\right)$.

Many authors $[30,31]$ computed the momentum exchange coefficient using

$\beta=\left(\rho_{\mathrm{p}}-\rho_{\mathrm{f}}\right) g \alpha_{\mathrm{p}} \alpha_{\mathrm{f}}^{(2-n)} / u_{\mathrm{T}}$.

where $n$ is the Richardson-Zaki exponent which depends on the particle settling Reynolds number $R e_{\mathrm{T}}=d_{\mathrm{p}} u_{\mathrm{T}} \rho_{\mathrm{f}} / \mu_{\mathrm{f}}$. Some authors [32-34] used the combined equations proposed by Ergun [35] when $\alpha_{\mathrm{f}} \leq 0.8$ and Wen and $\mathrm{Yu}$

\footnotetext{
${ }^{1}$ Bouyancy is one part of the inter-phase force.
} 
[36] when $\alpha_{\mathrm{f}}>0.8$ to compute the momentum exchange coefficient

$\beta=\left\{\begin{array}{ll}\mu_{\mathrm{f}} \alpha_{\mathrm{p}} d_{\mathrm{p}}^{-2} \alpha_{\mathrm{f}}^{-1}\left[150 \alpha_{\mathrm{p}}+1.75 R e\right] & \left(\alpha_{\mathrm{f}} \leq 0.8\right) \\ \frac{3}{4} C_{\mathrm{D}} \mu_{\mathrm{f}} \alpha_{\mathrm{p}} d_{\mathrm{p}}^{-2} \alpha_{\mathrm{f}}^{-2.7} R e & \left(\alpha_{\mathrm{f}}>0.8\right)\end{array}\right.$,

where $C_{\mathrm{D}}$ is the drag force coefficient for a single particle,

$C_{\mathrm{D}}= \begin{cases}24\left(1+0.15 R e^{0.687}\right) / R e & (\operatorname{Re} \leq 1000) \\ 0.44 & (R e>1000)\end{cases}$

and the Reynolds number is defined as $R e=d_{\mathrm{p}} \rho_{\mathrm{f}} \alpha_{\mathrm{f}} \mid$ $u_{\mathrm{f}}-u_{\mathrm{p}} \mid \mu_{\mathrm{f}}^{-1}$. Researches on the virtual mass force $F_{\mathrm{vm}}$ are much scarce as compared with those on the drag force. The various published expressions about the virtual mass force are not checked enough yet. Drew et al. [37] have proposed the following expression for the virtual mass force:

$$
\begin{aligned}
F_{\mathrm{vm}}= & -C_{\mathrm{vm}} \alpha_{p} \rho_{\mathrm{f}}\left[\frac{\partial u_{\mathrm{p}}}{\partial t}+u_{\mathrm{f}} \frac{\partial u_{\mathrm{p}}}{\partial x}-\frac{\partial u_{\mathrm{f}}}{\partial t}-u_{\mathrm{p}} \frac{\partial u_{\mathrm{f}}}{\partial x}\right. \\
& \left.-\left(1-\lambda_{\mathrm{vm}}\right)\left(u_{\mathrm{f}}-u_{\mathrm{p}}\right) \frac{\partial\left(u_{\mathrm{f}}-u_{\mathrm{p}}\right)}{\partial x}\right],
\end{aligned}
$$

where the model parameters $C_{\mathrm{vm}}$ and $\lambda_{\mathrm{vm}}$ are the functions of $\alpha_{\mathrm{p}}$, and $0<C_{\mathrm{vm}}<0.5,0<\lambda_{\mathrm{vm}}<2$. Some authors [29] using the following expression for the virtual mass force:

$F_{\mathrm{vm}}=-C_{\mathrm{vm}} \alpha_{\mathrm{p}} \rho_{\mathrm{f}}\left[\frac{\partial u_{\mathrm{p}}}{\partial t}+u_{\mathrm{p}} \frac{\partial u_{\mathrm{p}}}{\partial x}-\frac{\partial u_{\mathrm{f}}}{\partial t}-u_{\mathrm{f}} \frac{\partial u_{\mathrm{f}}}{\partial x}\right]$.

Generally speaking, the virtual mass force is in direct proportion of the relative acceleration between the two phases; however, there exist many expressions due to the different understandings about the relative acceleration [38].

Any kind of particle random motion induces momentum transfer. The solid phase pressure $p_{\mathrm{p}}$ and the normal stress $\tau_{\mathrm{p}, \mathrm{xx}}$ are the representations of such momentum transfer. ${ }^{2}$ The particle random motion arises from the turbulent fluctuations of the fluid and the collisions between particles. More and more attentions have been drawn to the study on solid phase pressure $p_{\mathrm{p}}$ and normal stress $\tau_{\mathrm{p}, \mathrm{xx}}$ because it is the main factor determining the stability of the flow in fluidized beds [1,39]. However, various expressions about them greatly differ from each other, indicating the necessity to study further. The solid phase pressure $p_{\mathrm{p}}$ is usually thought to be in relation to the local particle concentration $\alpha_{\mathrm{p}}$. Needham and Merkin [1], Homsy, et al. [40], Harris and Crighton [41] regarded $p_{\mathrm{p}}$ is a monotonously increasing function of $\alpha_{\mathrm{p}}$. Needham and Merkin [1] regarded $p_{\mathrm{p}}$ is the linear function of particle concentration $\alpha_{\mathrm{p}}, p_{\mathrm{p}}=p_{0} \alpha_{\mathrm{p}}$, where $p_{0}$ is a constant. In order to prevent $\alpha_{\mathrm{p}}$ from being larger than the packed

\footnotetext{
${ }^{2}$ In fact, both $p_{\mathrm{p}}$ and $\tau_{\mathrm{p}, \mathrm{xx}}$ belong to the stress of solid phase. The spherical part of the stress tensor which depends on particle concentration $\alpha_{\mathrm{p}}$ is usually called pressure $p_{\mathrm{p}}$ and the remainder which depends on the gradient of solid phase velocity is called partial stress tensor. $\tau_{\mathrm{p}, \mathrm{xx}}$ is a component of the partial stress tensor.
}

concentration $\alpha_{\mathrm{p}, \mathrm{c}}\left(\alpha_{\mathrm{p}, \mathrm{c}} \approx 0.6137\right)$, Harris and Crighton [41] proposed the following expression:

$p_{\mathrm{p}}=p_{0} \frac{\alpha_{\mathrm{p}}}{\alpha_{\mathrm{p}, \mathrm{c}}-\alpha_{\mathrm{p}}}$.

Batchelor [39] did not think $p_{\mathrm{p}}$ is a monotonously increasing function of $\alpha_{\mathrm{p}}$, and the expression for $p_{\mathrm{p}}$ has an extremum in a medium concentration. In the uniform fluidized state, $p_{\mathrm{p}}$ can be expressed as

$p_{\mathrm{p}}=\alpha_{\mathrm{p}} \rho_{\mathrm{p}} H\left(\alpha_{\mathrm{p}}\right) U^{2} \approx \rho_{\mathrm{p}} \frac{\alpha_{\mathrm{p}}^{2}}{\alpha_{\mathrm{p}, \mathrm{c}}}\left(1-\frac{\alpha_{\mathrm{p}}}{\alpha_{\mathrm{p}, \mathrm{c}}}\right) U^{2}$.

Zenit et al. [42] measured the solid phase pressure using the high frequency piezoelectricity transducer in a solidliquid system and compared the experimental results with many published approximate expressions for $p_{\mathrm{p}}$. It is shown that various expressions greatly depart from the experimental results except for Eq. (13). Recently, Duru et al. [29] have studied the concentration wave shape induced by the instability of the solid-liquid flow and derived the derivative of $p_{\mathrm{p}}: \mathrm{d} p_{\mathrm{p}} / \mathrm{d} \alpha_{\mathrm{p}}=0.7 \rho_{\mathrm{f}} u_{\mathrm{T}}^{2}$ or $\mathrm{d} p_{\mathrm{p}} / \mathrm{d} \alpha_{\mathrm{p}}=0.2 \rho_{\mathrm{f}} d u_{\mathrm{T}}^{2}$. These expressions differ from Eq. (13). Therefore, it is necessary to further study the model for $p_{\mathrm{p}}$. A modified relationship of Duru et al. [29] is used in the simulation in this study

$p_{\mathrm{p}}=s \rho_{\mathrm{p}} u_{\mathrm{T}}^{2} \alpha_{\mathrm{p}} /\left(\alpha_{\mathrm{p}, \mathrm{c}}-\alpha_{\mathrm{p}}\right)$.

where $s$ is an adjustable parameter.

The solid phase stress $\tau_{\mathrm{p}, \mathrm{xx}}$ can be expressed as [28,29]

$\tau_{\mathrm{p}, \mathrm{xx}}=\frac{3}{4} \mu_{\mathrm{p}} \frac{\partial u_{\mathrm{p}}}{\partial x}$.

Both the theoretical and experimental results about the solid phase viscous coefficient $\alpha_{p}$ are imperfect. Batchelor [39] used the expression: $\mu_{\mathrm{p}}=\beta_{\mu} d_{\mathrm{p}} \alpha_{\mathrm{p}} \rho_{\mathrm{p}} U$, where $\beta_{\mu}$ is the model parameter with the order of unit and varies with $\alpha_{\mathrm{p}}$. Anderson et al. [28] and Glasser et al. [31] used the expression $\mu_{\mathrm{p}}=A \alpha_{\mathrm{p}} /\left[1-\left(\alpha_{\mathrm{p}} / \alpha_{\mathrm{p}, \mathrm{c}}\right)^{1 / 3}\right]$ to study the instability of fluidized beds, where $A$ is a model parameter with a dimension of viscosity. Duru et al. [29] obtained the expression of $\mu_{\mathrm{p}}$ by experiments

$\mu_{\mathrm{p}}=C_{\mu} \rho_{\mathrm{p}} d_{\mathrm{p}} u_{\mathrm{T}}\left(\alpha_{\mathrm{p}, \mathrm{c}}-\alpha_{\mathrm{p}}\right)^{-1}$,

where $C_{\mu}$ is 0.18 in their study.

The above equation set Eqs. (1)-(5) including the $p_{\mathrm{p}}$, $F_{\mathrm{D}}, F_{\mathrm{vm}}$ and $\tau_{\mathrm{p}, \mathrm{xx}}$ terms is named the General Two-Fluid Model (GTFM) in this study.

For a constant fluidizing velocity $U$, when the flow in the bed is in a steady and uniform state, $\partial \varphi / \partial t=0$ and $\partial \varphi /$ $\partial x=0$ for an arbitrary variable $\varphi$ (except for pressure $p$ ). Under such circumstances, $F_{\mathrm{vm}}=0, F_{\mathrm{p}}=F_{\mathrm{D}}=\beta\left(u_{\mathrm{f}}-u_{\mathrm{p}}\right)$ and Eq. $\left(3^{\prime}\right)$ can be simplified as

$u_{\mathrm{f}}-u_{\mathrm{p}}=\alpha_{\mathrm{p}} \alpha_{\mathrm{f}}\left(\rho_{\mathrm{p}}-\rho_{\mathrm{f}}\right) g / \beta$. 
Introducing Eqs. (8) and (2') into Eq. (17) yields

$U-u_{\mathrm{p}}=\alpha_{\mathrm{f}}\left(u_{\mathrm{f}}-u_{\mathrm{p}}\right)=u_{\mathrm{T}} \alpha_{\mathrm{f}}^{n}$.

Because $\partial u_{\mathrm{p}} / \partial x=0$ and $\left.u_{\mathrm{p}}\right|_{x=0}=0$ in a steady and uniform state, it is concluded that $u_{\mathrm{p}}(x)=0$ everywhere in the bed, Eq. (18) becomes the well-known Richardson-Zaki relationship, $U=u_{\mathrm{T}} \alpha_{\mathrm{f}}^{n}$ [43]. Richardson-Zaki relationship has been demonstrated by even earlier fluidized bed experiments [44]. More recently, the relationship has been verified using direct numerical simulation [45]. It is proper to use Eq. (8) to express the drag force in fluidized beds. Introducing the Ergun relationship of Eq. (9) into Eq. (17) yields $U-u_{\mathrm{p}}=$ $\alpha_{\mathrm{f}}\left(u_{\mathrm{f}}-u_{\mathrm{p}}\right)=\left(\rho_{\mathrm{p}}-\rho_{\mathrm{f}}\right) g d_{\mathrm{p}}^{2} \mu_{\mathrm{f}}^{-1} \alpha_{\mathrm{f}}^{3}\left(150 \alpha_{\mathrm{p}}+1.75 R e\right)^{-1}$.

Our experimental results show that Richardson-Zaki relationship is more precise than Ergun relationship (Eq. (9)) when used to express the drag force (see Fig. 2) under the experimental conditions. The open points in Fig. 2 was gotten by a series of steady fluidization experiments (see Section 4), the results of Richardson-Zaki relationship and Ergun relationship are also given in the figure.

Since the constitutive relationships for $p_{\mathrm{p}}, F_{\mathrm{vm}}$ and $\tau_{\mathrm{p}, \mathrm{xx}}$ are still open now, neglecting these terms in Eqs. (3) and (4), one can simplify Eqs. (1)-(5) and get the inviscid flow equations with the drag force only remained in the interphase force between the phases, which is named the Basic Two-Fluid Model (BTFM) in this study. BTFM has been used since earlier researches. Recently, a multi-dimensional simulation of fluidization using this version of Two-Fluid Model has been performed by Zhang and VanderHeyden [46]. Although the equations in BTFM are simple, yet not without shortcomings, in particular, the eigenvalues of BTFM are complex numbers

$\lambda_{1,2}=\frac{\alpha_{\mathrm{f}} \rho_{\mathrm{p}} u_{\mathrm{p}}+\alpha_{\mathrm{p}} \rho_{\mathrm{f}} u_{\mathrm{f}}}{\alpha_{\mathrm{f}} \rho_{\mathrm{p}}+\alpha_{\mathrm{p}} \rho_{\mathrm{f}}} \pm i \frac{\sqrt{\alpha_{\mathrm{f}} \alpha_{\mathrm{p}} \rho_{\mathrm{p}} \rho_{\mathrm{f}}}}{\alpha_{\mathrm{f}} \rho_{\mathrm{p}}+\alpha_{\mathrm{p}} \rho_{\mathrm{f}}}\left|u_{\mathrm{f}}-u_{\mathrm{p}}\right|$,

thus making the equation set be ill-posed for initial-value problems. Complex eigenvalues indicate linear instability; small disturbances added to a constant solution of the

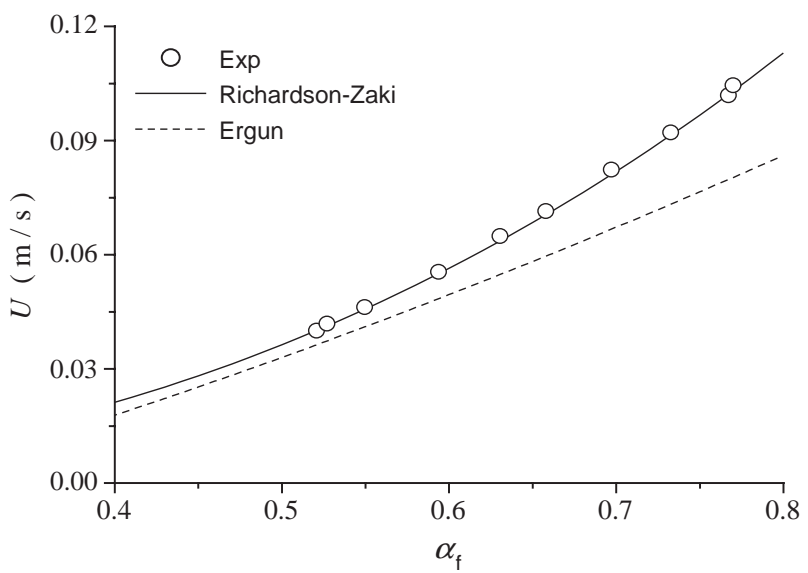

Fig. 2. Comparison between the Richardson-Zaki and Ergun relationship with the experimental results. system grow large rather than damp. For more details, please refer to $[22,47,48]$. The instability always existing in an ill-posed system suggests that the model is not treating small-scale phenomena correctly. Eq. (19) indicates that the larger the relative velocity of the two phases, the larger the imaginary part of the eigenvalues and the more unstable the equation set. Generally speaking, any initial small disturbance is enlarged in its traveling process for an ill-posed equation set. However, it may not be so serious in practical numerical simulation. The numerical viscosity produced by discretizating the partial differential equations suppresses numerical oscillations, for example, the simulation performed in $[15,46]$ and the simulation of Case A in Section 5 of this paper. If the pulse height of fluidizing velocity $\Delta U$ ( $=U_{2}-U_{1}, U_{2}$ and $U_{1}$ are fluid superficial velocity during the semi-on period $T_{2}$ and semi-off period $T_{1}$, respectively, see Fig. 3a) is too large, however, the numerical oscillations grow quickly and computation fails, for example, the simulation of Case B in Section 5.

If Eqs. (14) and (11) for $p_{\mathrm{p}}$ and $F_{\mathrm{vm}}$ are introduced in Eqs. (3) and (4), the eigenvalues of the equation set Eqs. (1)-(5) are

$\lambda_{1,2}=\frac{\rho_{1} u_{\mathrm{p}}+\rho_{2} u_{\mathrm{f}} \pm \sqrt{\alpha_{\mathrm{f}}\left(\rho_{1}+\rho_{2}\right) G-\rho_{1} \rho_{2}\left(u_{\mathrm{f}}-u_{\mathrm{p}}\right)^{2}}}{\rho_{1}+\rho_{2}}$,

where $\rho_{1}=\alpha_{\mathrm{f}}\left(\rho_{\mathrm{p}}+\rho_{\mathrm{f}} C_{\mathrm{vm}} / \alpha_{\mathrm{f}}\right) ; \quad \rho_{2}=\alpha_{\mathrm{p}} \rho_{\mathrm{f}}\left(1+C_{\mathrm{vm}} / \alpha_{\mathrm{f}}\right)$; $G=\partial p_{\mathrm{p}} / \partial \alpha_{\mathrm{p}}=s \rho_{\mathrm{p}} u_{\mathrm{T}}^{2} \alpha_{\mathrm{p}, \mathrm{c}}\left(\alpha_{\mathrm{p}, \mathrm{c}}-\alpha_{\mathrm{p}}\right)^{-2}$. If $G$ is large enough, GTFM becomes hyperbolic.

The boundary and initial conditions are:

$$
\begin{aligned}
& x=0: u_{\mathrm{p}}(t, 0)=0, \quad \alpha_{\mathrm{p}}(t, 0)=1-\left(U(t) / u_{\mathrm{T}}\right)^{1 / n} ; \\
& x=h(t): u_{\mathrm{p}}(t, h)=\mathrm{d} h(t) / \mathrm{d} t ;
\end{aligned}
$$

$t=0: \alpha_{\mathrm{p}}(0, x)=\alpha_{\mathrm{p}, 0}, \quad u_{\mathrm{p}}(0, x)=0, \quad h(0)=h_{0}$.

where a uniform fluidized state is assumed at $t=0, \alpha_{\mathrm{p}, 0}$ and $h_{0}$ are the initial particle concentration and bed height respectively, $h(t)$ is the instantaneous bed height, $u_{\mathrm{p}}(t, h)$ is the instantaneous particle velocity at the bed surface.

The first order forward finite difference scheme and a five order WENO (Weighted Essentially Non-Oscillatory) scheme [49] are used to solve BTFM and GTFM respectively. The numerical results compared with experimental results are shown in Section 5. Our numerical experiments show that, under certain conditions, especially when the pulse height of fluidizing velocity $\Delta U$ is large, the simulation using BTFM often fails unless a large numerical viscosity (corresponding to a relative coarse grid) is used to suppress the unstable solutions, while the numerical solution of GTFM is always convergent only if $G$ is large enough.

Although the inclusion of $p_{\mathrm{p}}$ and $F_{\mathrm{vm}}$ in GTFM can make it well-posed, the understanding of the constitutive relationship of $p_{\mathrm{p}}, F_{\mathrm{vm}}$ and $\tau_{\mathrm{p}, \mathrm{xx}}$ are not complete now, and 

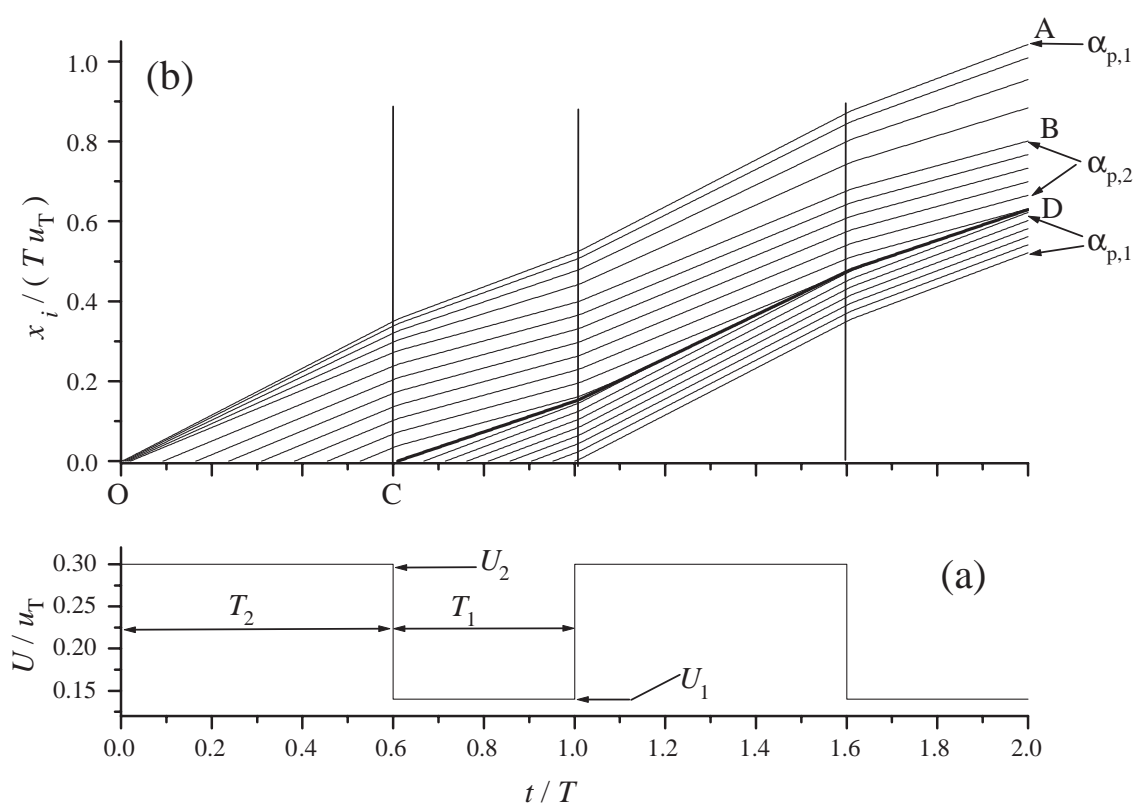

Fig. 3. Diagram of the characteristic lines when $U(t)$ varies with time as a rectangular wave.

the adjustable parameters in these expressions cannot be accurately determined beforehand and are chosen at will to some extent. In order to grasp the main characteristic of the pulsed flow in the bed, we intend to propose a simplified model of BTFM, in which there are no adjustable parameters, to avoid the instability caused by its ill-posed property.

\section{Local equilibrium model (LEM)}

There are many approximate methods to avoid the complex eigenvalues. For example, neglecting the second term on the left hand side of Eq. $\left(3^{\prime}\right)$, one can get

$\rho_{\mathrm{p}}\left(\frac{\partial u_{\mathrm{p}}}{\partial t}+u_{\mathrm{p}} \frac{\partial u_{\mathrm{p}}}{\partial x}\right)=\frac{F_{\mathrm{D}}}{\alpha_{\mathrm{p}} \alpha_{\mathrm{f}}}-\left(\rho_{\mathrm{p}}-\rho_{\mathrm{f}}\right) g-\frac{1}{\alpha_{\mathrm{p}}} \frac{\partial p_{\mathrm{p}}}{\partial x}$.

Eq. (24) corresponds to the solid phase momentum equation of the Model $\boldsymbol{B}$ in Gidaspow's book [20] and the eigenvalues become

$\lambda_{1,2}=u_{\mathrm{p}} \pm \sqrt{G / \rho_{\mathrm{p}}}$.

For small value of $G$, the eigenvalues are nearly equal. Particles essentially move with their own velocity, with the wave effect in the bed negligible. Particularly, if the value of $G$ equals zero, then $\lambda_{1,2}=u_{\mathrm{p}}$ and the eigenvalues are equal, thus making the model be still unstable to small disturbance according to the theory of Wallis [50]. ${ }^{3}$

\footnotetext{
3 Though the eigenvalues are real, the speed of the dynamic wave is zero and it is smaller than the kinematic wave speed.
}

Another method is that both terms on the left hand side of Eq. $\left(3^{\prime}\right)$ are neglected based on BTFM and the first order partial differential equation degenerates into an algebraic relationship

$0=F_{\mathrm{D}}-\left(\rho_{\mathrm{p}}-\rho_{\mathrm{f}}\right) \alpha_{\mathrm{p}} \alpha_{\mathrm{f}} g$.

In Eq. (26), the relative motion between the two phases is fully determined by the transient balance between the interphase drag force $F_{\mathrm{D}}$ and the gravity force (corrected by the buoyancy) per unit mixture volume, $-\alpha_{\mathrm{p}} \rho_{\mathrm{p}} g+\alpha_{\mathrm{p}}\left(\alpha_{\mathrm{p}} \rho_{\mathrm{p}}+\right.$ $\left.\alpha_{\mathrm{f}} \rho_{\mathrm{f}}\right) g$, where $-\alpha_{\mathrm{p}} \rho_{\mathrm{p}} g$ is the gravity force of the particles in that volume and $\alpha_{\mathrm{p}}\left(\alpha_{\mathrm{p}} \rho_{\mathrm{p}}+\alpha_{\mathrm{f}} \rho_{\mathrm{f}}\right) g$ is buoyancy to the particles produced by the surrounding liquid-solid mixture. Introducing Eqs. (7) and (8) into Eq. (26) yields

$U(t)-u_{\mathrm{p}}(t, x)=u_{\mathrm{T}}\left(1-\alpha_{\mathrm{p}}(t, x)\right)^{n}$.

There are no partial derivatives of the variables to time $t$ and space coordinate $x$ in the above equation, it is the local equilibrium equation among the fluidizing velocity $U$, particle velocity $u_{\mathrm{p}}$ and particle volume fraction $\alpha_{\mathrm{p}}$, thus it can be named the Local Equilibrium Model (LEM).

The above two methods, ignoring either one term or the difference of the two terms on the left hand side of Eq. $\left(3^{\prime}\right)$, are the approximations of the original model. It is difficult to judge which method introduces a smaller error for the dense liquid-solid flow. Eq. (27) is much simpler than Eq. (24) and clearly describes the wave characteristic of the flow in the bed (see Eq. (28)). Therefore, we would choose Eq. (27) to model the momentum transfer in the pulsed bed.

Many authors have used Eq. (27) as a direct generalization of the Richardson-Zaki relationship [43]. For example, Slis et al. [9] have studied the response of the bed height of a liquid fluidized bed to a sudden change of fluidizing velocity. 
Introducing Eq. (27) into Eq. (1) and eliminating particle velocity $u_{\mathrm{p}}$ one can get a hyperbolic concentration wave equation

$\frac{\partial \alpha_{\mathrm{p}}}{\partial t}+V \frac{\partial \alpha_{\mathrm{p}}}{\partial x}=0$

where $V$ is the concentration wave speed and the eigenvalue of the equation

$$
\begin{aligned}
\lambda & =V=\frac{\partial\left(\alpha_{\mathrm{p}} u_{\mathrm{p}}\right)}{\partial \alpha_{\mathrm{p}}}=u_{\mathrm{p}}+\alpha_{\mathrm{p}} \frac{\partial u_{\mathrm{p}}}{\partial \alpha_{\mathrm{p}}} \\
& =U(t)+\left[n \alpha_{\mathrm{p}}\left(1-\alpha_{\mathrm{p}}\right)^{n-1}-\left(1-\alpha_{\mathrm{p}}\right)^{n}\right] u_{\mathrm{T}} .
\end{aligned}
$$

For a given fluidizing velocity $U_{1}$ to $U_{2}$, the concentration wave speed $V$ has a maximum at $\alpha_{\mathrm{p}}=\alpha_{\mathrm{p}, \mathrm{cr}}=2 /(n+1)$. Under our experimental conditions $n=2.414$, and $a_{\mathrm{p}, \mathrm{cr}}=$ $0.5858 \approx a_{\mathrm{p}, \mathrm{c}}$, thus $V$ usually increases with the increase of $\alpha_{\mathrm{p}}$.

When the fluidizing velocity $U$ suddenly increases from $U_{1}$ to $U_{2}$, a disturbance with finite amplitude, $\left(\Delta \alpha_{\mathrm{p}}\right)_{\exp }=$ $\alpha_{\mathrm{p}, 2}-\alpha_{\mathrm{p}, 1}<0$ (where $\alpha_{\mathrm{p}, 2}=1-\left(U_{2} / u_{\mathrm{T}}\right)^{1 / \mathrm{n}}, \alpha_{\mathrm{p}, 1}=1-\left(U_{1} /\right.$ $\left.\left.u_{\mathrm{T}}\right)^{1 / \mathrm{n}}\right)$, is introduced at the bottom of the bed. This finite disturbance can be regarded as the accumulation of many infinitesimal disturbances. Because $\alpha_{\mathrm{p}, 1}>\alpha_{\mathrm{p}, 2}$ and the disturbances in the upper section with high particle concentration travel faster than the disturbances in the lower section with dilute particle concentration, i.e., $V\left(\alpha_{\mathrm{p}, 1}\right)>V\left(\alpha_{\mathrm{p}, 2}\right)$, the transitional section from $\alpha_{\mathrm{p}, 1}$ in the upper section to $\alpha_{\mathrm{p}, 2}$ in the lower section broadens when it travels upward in the bed.

By contraries, when the fluidizing velocity $U$ suddenly decreases from $U_{2}$ to $U_{1}$ a disturbance with finite amplitude, $\left(\Delta \alpha_{\mathrm{p}}\right)_{\mathrm{coll}}=\alpha_{\mathrm{p}, 1}-\alpha_{\mathrm{p}, 2}>0$, is introduced at the bottom of the bed. This finite disturbance can also be regarded as the accumulation of many infinitesimal disturbances. The disturbances in the lower section with high concentration travel faster and overtake the disturbances in the upper section with dilute particle concentration, and the transition section from $\alpha_{\mathrm{p}, 2}$ in the upper section to $\alpha_{\mathrm{p}, 1}$ in the lower section thus remains sharp when it travels upwards in the bed. A concentration discontinuity (or shock wave) occurs in the collapse process.

The above behaviors of concentration wave in the fluidized bed are very similar to those of the dynamic waves in a shock tube (or a pipe with a piston) in gas dynamics. There exist dilatation wave, compression wave and shock wave in both the pulsed fluidized bed and the shock tube. For example, in a pipe with a piston moving right at a velocity of $U_{\text {pis}}$, the gas before the piston flows at the same velocity as the piston. If the piston velocity suddenly decreases from $U_{\mathrm{pis}, 2}$ to $U_{\mathrm{pis}, 1}\left(U_{\mathrm{pis}, 2}>U_{\mathrm{pis}, 1}\right)$ at the time $t=t_{0}$, a series of dilatation waves occurs in front of the piston and propagates right (corresponding to the dilatation waves on the upside of the distributor during expansion process in fluidized bed), followed by a stable state flowing at the velocity of $U_{\text {pis, } 1 .}$. On the contrary, if the piston velocity suddenly increases from
$U_{\mathrm{pis}, 1}$ to $U_{\mathrm{pis}, 2}\left(U_{\mathrm{pis}, 1}<U_{\mathrm{pis}, 2}\right)$ at $t=t_{0}$, a series of compression waves occurs before the piston and propagates right. Because the speed of the compression wave arising earlier is slower than that arising later, the later will catch up with the earlier and they will aggregate into a dynamic shock.

Let $V_{s}$ be the speed of the concentration shock. Integrating Eq. (1) across the shock wave yields the shock conservative condition

$V_{s}\left(\alpha_{\mathrm{p}, \mathrm{b}}-\alpha_{\mathrm{p}, \mathrm{f}}\right)=\alpha_{\mathrm{p}, \mathrm{b}} u_{\mathrm{p}, \mathrm{b}}-\alpha_{\mathrm{p}, \mathrm{f}} u_{\mathrm{p}, \mathrm{f}}$.

Substituting Eq. (27) into Eq. (30) yields

$$
\begin{aligned}
V_{s} & =\frac{\alpha_{\mathrm{p}, \mathrm{b}} u_{\mathrm{p}, \mathrm{b}}-\alpha_{\mathrm{p}, \mathrm{f}} u_{\mathrm{p}, \mathrm{f}}}{\alpha_{\mathrm{p}, \mathrm{b}}-\alpha_{\mathrm{p}, \mathrm{f}}} \\
& =U(t)-\frac{\alpha_{\mathrm{p}, \mathrm{b}}\left(1-\alpha_{\mathrm{p}, \mathrm{b}}\right)^{n}-\alpha_{\mathrm{p}, \mathrm{f}}\left(1-\alpha_{\mathrm{p}, \mathrm{f}}\right)^{n}}{\alpha_{\mathrm{p}, \mathrm{b}}-\alpha_{\mathrm{p}, \mathrm{f}}} u_{\mathrm{T}},
\end{aligned}
$$

where $\alpha_{\mathrm{p}, \mathrm{b}}, \alpha_{\mathrm{p}, \mathrm{f}}$ are the particle volume fraction at the back and front of the shock and $u_{\mathrm{p}, \mathrm{b}}, u_{\mathrm{p}, \mathrm{f}}$ are the particle velocity at the back and front of the shock respectively.

Eq. (28) is hyperbolic and avoids the divergence of numerical simulation caused by the ill-posed property of BTFM. In this study, we use the method of characteristics to solve Eqs. (28) and (30). At the very beginning of an expansion process, a series of dilatation waves occurs on the upside of the distributor, every dilatation wave corresponds to a line with a slope of $V$ (see Eq. (29)) on the $x-t$ plane. According to Eq. (28), particle concentration keeps a constant along the corresponding characteristic line. Fluidized state gradually expands from particle concentration $\alpha_{\mathrm{p}, 1}$ to particle concentration $\alpha_{\mathrm{p}, 2}$, the dilatation waves distribute in the fan $A O B$ on the $x-t$ plane, whose upper boundary is the line with a slope of $V\left(\alpha_{\mathrm{p}, 1}\right)$ and lower boundary is the line with a slope of $V\left(\alpha_{\mathrm{p}, 2}\right)$ and $V\left(\alpha_{\mathrm{p}, 2}\right)<V\left(\alpha_{\mathrm{p}, 1}\right)$. In practical computation, we use finite times $(N)$ of expansion (corresponding to $N$ characteristic lines on the $x-t$ plane) to represent the continuous expansion from $\alpha_{\mathrm{p}, 1}$ to $\alpha_{\mathrm{p}, 2}$ (see the thin lines in the fan $A O B$ in Fig. 3b). At the beginning of a collapse process, a series of compression waves from $\alpha_{\mathrm{p}, 2}$ to $\alpha_{\mathrm{p}, 1}$ occurs on the upside of the distributor. The compression waves aggregate into a discontinuity of particle concentration (concentration shock wave) with a shock speed $V_{s}$ that meets the shock conservative condition Eq. (30). We use the shock line to replace these characteristic lines, (see the thick line $C D$ Fig. $3 \mathrm{~b}$ ). After the fluidizing velocity $U(t)$ suddenly changes each time, the slope of every characteristic line and the shock wave line also change, see Eqs. (29) and $\left(30^{\prime}\right)$.The numerical results are shown in Section 5.

\section{Experimental apparatus and method}

\subsection{Experimental apparatus}

Fig. 4 is the schematic diagram of the experimental facility. The experiments on solid-liquid pulsed fluidization 


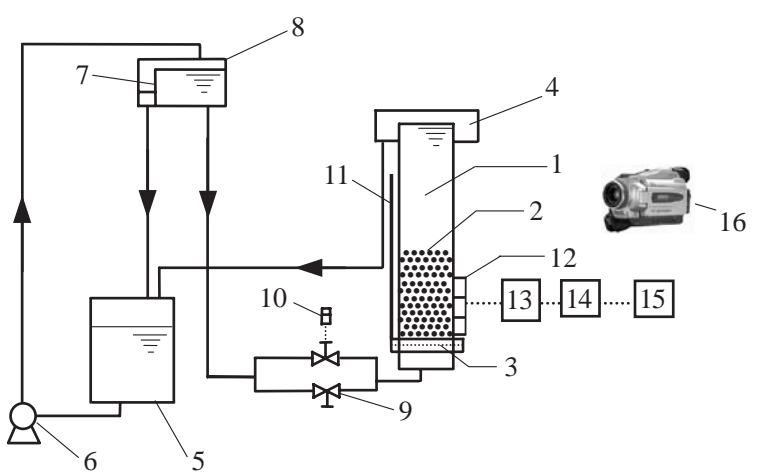

Fig. 4. Schematic diagram of experimental facility of pulsed fluidized bed. (1) fluidized bed; (2) glass beads; (3) distributor; (4) water gathering vessel; (5) lower water tank; (6) centrifugal pump; (7) overflow plate; (8) upper water tank; (9) ball valve; (10) time-delay relay and solenoid valve; (11) ruler; (12) pressure transducer; (13) amplifier; (14) A/D converter; (15) computer; (16) digital recorder.

were carried out in a 30-mm ID, 2-m high Plexiglas tube with a porous distributor (3) mounted at its bottom. Water from upper tank (8) flows down to fluidize the glass beads (2) in fluidized bed (1). The pulse frequency $(1 / T)$, pulse width (fraction of semi-on period in a whole period, i.e., $T_{2} /$ $T)$ and pulse height $\Delta U$ of the fluidizing velocity are controlled by a ball valve (9), two time-delay relays and a solenoid valve (10). When the solenoid valve is shut off, fluid flows only through the ball valve (9), and $U(t)$ in the bed (1) equals $U_{1}$; when the solenoid valve is turned on, water flows through both the ball valve (9) and the solenoid valve (10), and $U(t)$ in the bed (1) equals $U_{2}$ $\left(U_{2}>U_{1}>U_{\mathrm{mf}}\right)$. The time-delay relays control the solenoid valve on or off and $U(t)$ pulsates with time as a rectangular wave between $U_{2}$ and $U_{1}$ to impart pulsed fluidization in the bed (1). In order to reduce the non-uniformity of the inlet flow near the distributor, a section with packed small glass beads was mounted below the distributor (3) to uniformly distribute the liquid flow. The flow rate was measured using a platform balance and a stopwatch. The bed height $h(t)$ and the transient distribution of particle concentration $\alpha_{\mathrm{p}}(t, x)$ were recorded by a Sony ${ }^{\circledR}$ digital video recorder (16) (DCRTRV6E). The properties of particles used are shown in Table 1. The sketch map of fluidizing velocity vs. time is illustrated in Fig. 3a.

\subsection{Experimental method}

We put a ruler (11) beside the bed in the experiment and record the variational process of the bed height by the recorder (16). The recorder can record 25 frames per second.

Table 1

Physical properties of the particles and fluid

\begin{tabular}{lllllll}
\hline Particles & $d_{\mathrm{p}}(\mathrm{mm})$ & $\rho_{\mathrm{p}}\left(\mathrm{kg} / \mathrm{m}^{3}\right)$ & $u_{\mathrm{T}}(\mathrm{m} / \mathrm{s})$ & $n$ & $\tau_{\mathrm{p}}(\mathrm{s})$ & $\rho_{\mathrm{p}}\left(\mathrm{kg} / \mathrm{m}^{3}\right)$ \\
\hline Glass beads & 1.8 & 2600.0 & 0.1937 & 2.412 & 0.032 & 1000.0 \\
\hline
\end{tabular}

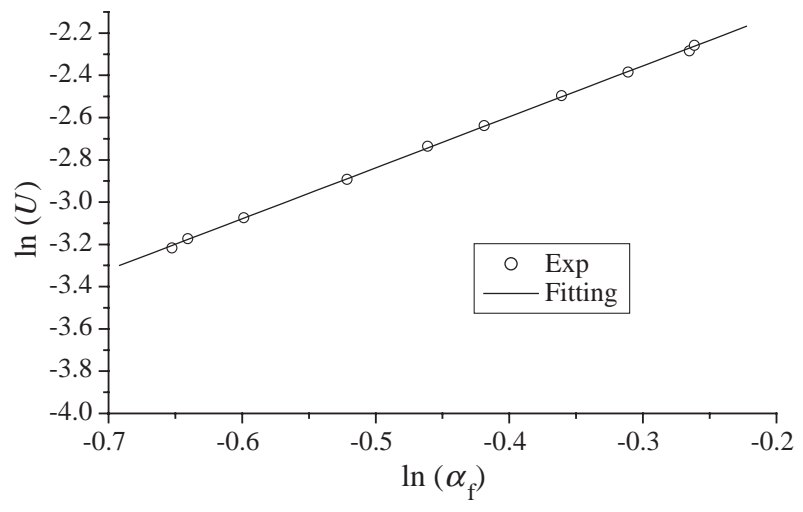

Fig. 5. Fitting the experimental data on fluidizing velocity and particle concentration.

The variation of bed height vs. time is read by playing the tape frame by frame. In order to get the settling velocity $u_{\mathrm{T}}$ and the Richardson-Zaki exponent $n$, we perform a series of steady fluidized experiments, recording the different bed height $h_{i}$ to get corresponding particle concentration $\alpha_{\mathrm{p}, i}$ at different fluidizing velocities $U_{i}$ using the particle conservative relationship $h_{\mathrm{p}, \mathrm{c}} \alpha_{\mathrm{p}, \mathrm{c}}=h_{i} \alpha_{\mathrm{p}, i}(i=1,2 \cdots)$, where $\alpha_{\mathrm{p}, \mathrm{c}}=0.6137$ is the packed particle concentration, $h_{\mathrm{p}, \mathrm{c}}$ is the bed height at the packed state and $\alpha_{\mathrm{f}, i}=1-\alpha_{\mathrm{p}, i}$. We fit the different data $\left(U_{i}\right.$ and $\left.\alpha_{\mathrm{f}, i}\right)$ using $U_{i}=u_{\mathrm{T}} \alpha_{\mathrm{f}, i}^{n}$ and get $n=2.414$ from the slope $B$, and the settling velocity $u_{\mathrm{T}}=0.1937$ from the intercept $A$ (see Fig. 5 and Table 2). The particle Reynolds number $R e_{\mathrm{T}}=348.7$. According to the relationship suggested by Richardson and Zaki [43], $n=4.4 R e_{\mathrm{T}}^{-0.1}=2.450$. The fitting result is very close to it.

\subsection{Measurement correction}

In order to measure the particle concentration distribution along the bed height, calibration must be performed to get the relationship between the gray scale of the digital photos and the particle concentration. We carried out the experiment at night to eliminate the effect of the variational environment light, and used six $500 \mathrm{~W}$-iodine-tungsten lamps in vertical direction to get uniform background light. We performed space corrections considering the nonuniform phototonus of the film at different points even to uniform light. For example, Fig. 6 shows that for a uniform state $\alpha_{\mathrm{p}}=0.2417$ throughout the bed, the gray scale of the photo is non-uniform along the bed height. After we do the nonlinear space corrections, we can get a much more uniform distribution of the particle concentration, the

Table 2

Fitting results of the coefficients $A$ and $B$ in Fig. 5

\begin{tabular}{lrl}
\hline Parameter & Value & Error \\
\hline$A$ & -1.641 & 0.00802 \\
$B(n)$ & 2.414 & 0.01702 \\
\hline $\operatorname{Ln}(U)=A+B \operatorname{Ln}\left(\alpha_{\mathrm{f}}\right)$. & &
\end{tabular}




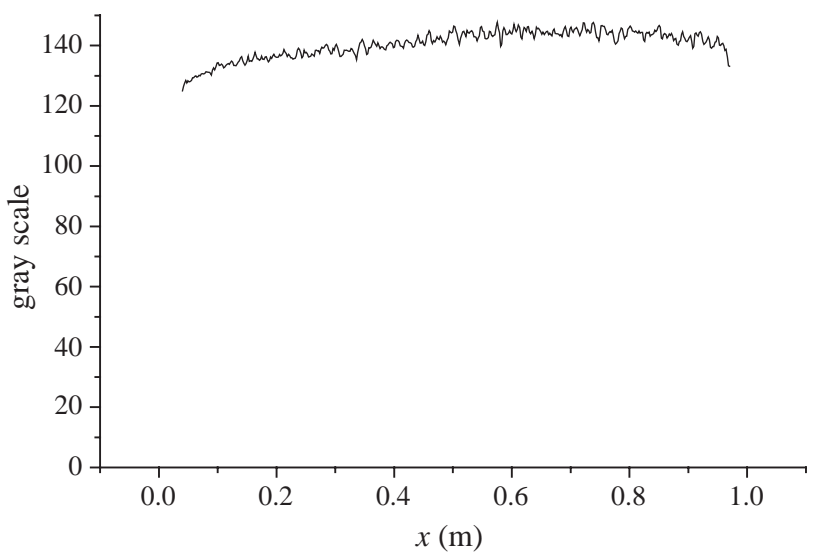

Fig. 6. Non-uniformity of the gray scale of the photo along the bed height for uniform concentration.

standard deviation is about $0.7 \%$ and the system error is about $1.0 \%$ (Fig. 7 ).

\section{Numerical results and comparison with experimental data}

In this section, we show the numerical results from GTFM, BTFM and LEM. We also give the experimental data to check the numerical simulation results. The test cases are listed in Table 3.

\subsection{Case A}

Fig. 8 shows the bed height variation with time responding to a periodically pulsed fluidizing velocity from GTFM, BTFM, LEM and the experimental data.

At the beginning of the pulsed process $\left(t<t_{\mathrm{A}}\right.$ in Fig. 8), the concentration disturbances from the distributor do not travel to the bed surface, the particle concentration at the bed surface is a constant and does not change with time, particle velocity at bed surface almost varies with the fluidizing velocity simultaneously ${ }^{4}$ according to Eq. (27). The slopes of the bed height before $t_{\mathrm{A}}$ are sectionwise constants with the change of the fluidizing velocity. The bed height fluctuates periodically after $t_{\mathrm{A}}$ according to LEM, while there is still a short transitional duration from $t_{\mathrm{A}}$ to the time when the bed height completely fluctuates periodically according to GTFM and BTFM.

Fig. 9 shows the non-uniform distribution of sectionaveraged particle concentration (a-f) and particle velocity $(\mathrm{g}-1)$ along the axial direction and the variation at different times in a period when the flow state in the bed is fully

\footnotetext{
${ }^{4}$ It is assumed that $\rho_{\mathrm{f}}=$ constant and $\rho_{\mathrm{p}}=$ constant, thus the fluid velocity $u_{\mathrm{f}}(t, h(t))$ and particle velocity $u_{\mathrm{p}}(t, h(t))$ at the bed surface simultaneously change with the fluidizing velocity $U(t) . U=U_{2}$, $h=h_{0}=1.37 \mathrm{~m}$ and $\mathrm{d} h / \mathrm{d} t=u_{\mathrm{p}}(t, h)=0$ when $t<0 ; U=U_{1}, \mathrm{~d} h /$ $\mathrm{d} t=u_{\mathrm{p}}(t, h)=U_{1}-U_{2}=-0.0512 \mathrm{~m} / \mathrm{s}$ when $0<\mathrm{t}<6 s$ and $U=U_{2}, \mathrm{~d} h /$ $\mathrm{d} t=u_{\mathrm{p}}(t, h)=0$ when $6<t<9 s$ (See Subsection 6.1 for details).
}

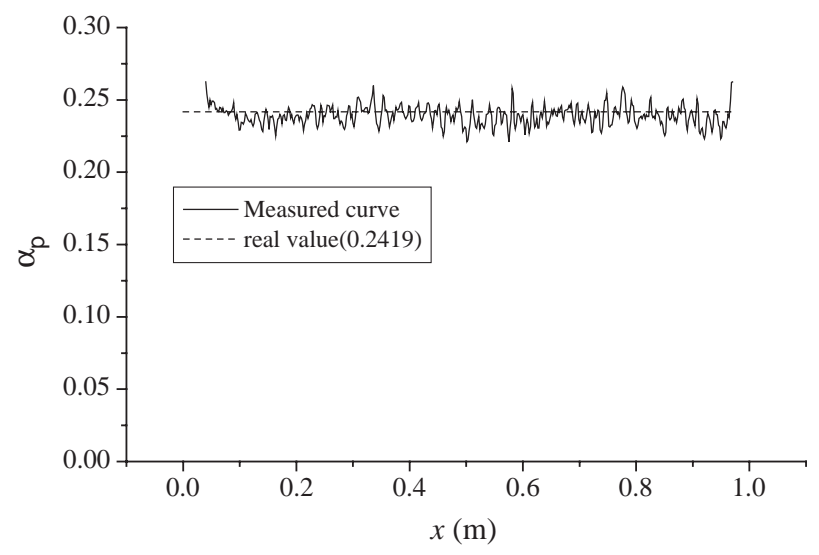

Fig. 7. Particle concentration along the bed after space correction of Fig. 6.

developed and periodical, where $t_{0}$ in the figure represents the beginning of one pulsed cycle.

The liquid volume fraction can be gotten from Eq. (5), $\alpha_{\mathrm{f}}=1-\alpha_{\mathrm{p}}$ and liquid velocity can be easily gotten from Eq. $\left(2^{\prime}\right), u_{\mathrm{f}}=\left(U(t)-\alpha_{\mathrm{p}} u_{\mathrm{p}}\right) / \alpha_{\mathrm{f}}$. Another unknown pressure $p$ can be gotten by integrating the Eq. $\left(4^{\prime}\right)$ along the axial direction.

From Figs. 8 and 9, one can see that the results gotten from GTFM, BTFM and LEM are similar and well match the experimental results with an acceptable accuracy.

\subsection{Case B}

In this case, the pulse height of fluidizing velocity $\Delta U\left(=U_{2}-U_{1}\right)$ is larger than that in Case $\mathrm{A}$, and the pulsed frequency is higher. The dynamic behaviors of this case are depicted photographically in Fig. 1. Fig. 10 shows the bed height variation with time responding to a pulsed fluidizing velocity from GTFM, LEM and the experimental data when the state is periodical. Fig. 11 shows the non-uniform distribution of section-averaged particle concentration $(a-f)$ and particle velocity $(\mathrm{g}-1)$ along the axial direction and the variation at different times in a period.

From Figs. 10 and 11, one can know that the numerical results obtained from GTFM and LEM also fit the experimental data well.

In Case A, GTFM, BTFM and LEM work successfully and the simulation using LEM saves much time compared with GTFM and BTFM. In Case B, we use a larger pulse height of fluidizing velocity $\Delta U$ than that in Case A, the simulation using BTFM is divergent due to its ill-posedness. Numerical oscillation occurs at the discontinuity of particle concentration where the particle concentration sharply

Table 3

Summary of the test cases

\begin{tabular}{llllll}
\hline & $U_{1}(\mathrm{~m} / \mathrm{s})$ & $U_{2}(\mathrm{~m} / \mathrm{s})$ & $T_{1}(\mathrm{~s})$ & $T_{2}(\mathrm{~s})$ & $h_{0}(\mathrm{~m})$ \\
\hline Case A & 0.055 & 0.1062 & 6.0 & 3.0 & 1.365 \\
Case B & 0.055 & 0.1482 & 3.0 & 1.0 & 1.950 \\
\hline
\end{tabular}



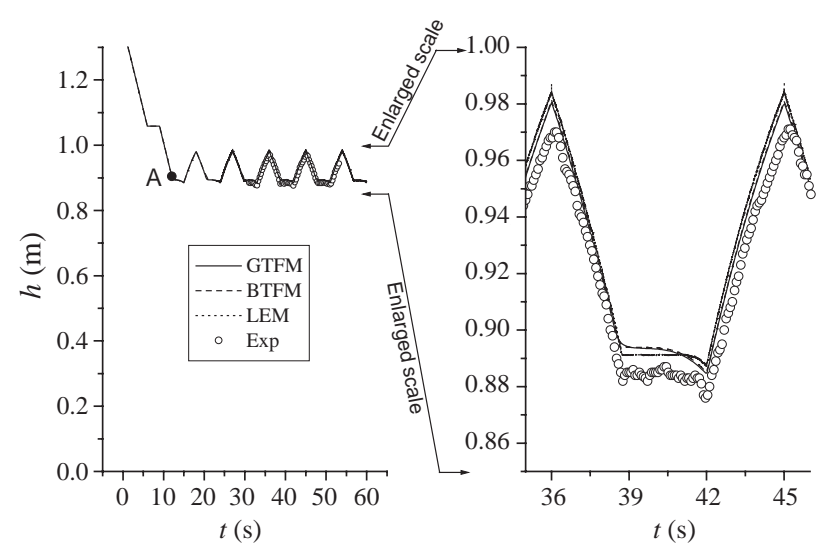

Fig. 8. Bed height variation with time responding to a periodically pulsed fluidizing velocity $\left(s=0.1, C_{\mu}=0, C_{\mathrm{vm}}=0.5\right.$ in GTFM).

changes. GTFM and LEM can simulate the condition well compared with the experimental data.

However, several undetermined constitutive relationships are included in GTFM, the adjustable parameters of which are not known prior and always chosen at will to some extent. Although there are no adjustable parameters in BTFM, its eigenvalues are complex numbers and it is ill-posed for initial-value problems. Ill-posedness causes divergence of the numerical results with grid refining, while numerical viscosity may introduce unacceptable errors with too coarse grids. Although LEM, a further simplification of BTFM, is very simple, it is highly capable of simulating complex processes in pulsed fluidization over a broad range of operating conditions, and its numerical results well fit experimental results in both the variation of bed height and the distribution of particle concentration as fluidizing velocity varies.

According to the experimental data, it is not true that the concentration discontinuity obtained from LEM is a plane without thickness as shown in Figs. 9 and 11. The limitation of LEM will be discussed at length in the next section.

\section{Shortcomings of LEM}

The above section shows that LEM is highly capable of simulating the complex processes of pulsed fluidization even though it is relatively simple. However, it has shortcomings. In this section, we will discuss the time scale that errors exist after a sudden change of fluidizing velocity and the spatial region where errors exist when sharp gradient of particle concentration exists in the flow field.

\subsection{Relaxation processes of the two-phase system after a sudden change of fluidizing velocity}

The uniform flow field in the bed is assumed in this subsection, i.e., $\partial / \partial x=0$. If the fluidizing velocity $U$ gets a sudden increment $\Delta U$ at $t=t_{0}$, the corresponding increments of $u_{\mathrm{p}}, u_{\mathrm{f}}, \alpha_{\mathrm{p}}$ and $\alpha_{\mathrm{f}}$ can be obtained from the Eqs. (1), ( $\left.2^{\prime}\right)$, (5) and (27): $\left(\Delta \alpha_{\mathrm{p}}\right)_{\mathrm{LEM}}=\left(\Delta \alpha_{\mathrm{f}}\right)_{\mathrm{LEM}}=0,\left(\Delta u_{\mathrm{p}}\right)_{\mathrm{LEM}}=$
$\left(\Delta u_{\mathrm{f}}\right)_{\mathrm{LEM}}=\Delta U$ according to LEM, where $\Delta \varphi=\varphi\left(t_{0+}\right)-$ $\varphi\left(t_{0-}\right), \varphi=\alpha_{\mathrm{p}}, \alpha_{\mathrm{f}}, u_{\mathrm{p}}, u_{\mathrm{f}}, U, \ldots \ldots$ In fact, the inertia of the two phases is different and it becomes very important when fluidizing velocity $U$ gets a jump change, the difference of the inertial forces between the two phases thus has considerable influence. The influence of inertia on the relaxation process after a jump change of $U$ is discussed hereinafter.

If the virtual mass is ignored, the increments $\Delta \alpha_{\mathrm{p}}, \Delta \alpha_{\mathrm{f}}$, $\Delta u_{\mathrm{p}}, \Delta u_{\mathrm{f}}$ can be obtained from BTFM, i.e., Eqs. (1), (2'), (5) and $\left(3^{\prime}\right)$ considering the different inertia between the two phases, (see Appendix). $\left(\Delta \alpha_{\mathrm{p}}\right)_{\mathrm{TFM}}=\left(\Delta \alpha_{\mathrm{f}}\right)_{\mathrm{TFM}}=0, \alpha_{\mathrm{p}}$ $\left(\Delta u_{\mathrm{p}}\right)_{\mathrm{TFM}}+\alpha_{\mathrm{f}}\left(\Delta u_{\mathrm{f}}\right)_{\mathrm{TFM}}=\Delta U, \rho_{\mathrm{p}}\left(\Delta u_{\mathrm{p}}\right)_{\mathrm{TFM}}-\rho_{\mathrm{f}}\left(\Delta u_{\mathrm{f}}\right)_{\mathrm{TFM}}=0$, therefore, $\left(\Delta u_{\mathrm{p}}\right)_{\mathrm{TFM}}=\left(\rho_{\mathrm{f}} \Delta U\right) /\left(\alpha_{\mathrm{p}} \rho_{\mathrm{f}}+\alpha_{\mathrm{f}} \rho_{\mathrm{p}}\right), \quad\left(\Delta u_{\mathrm{f}}\right)_{\mathrm{TFM}}=$ $\left(\rho_{\mathrm{p}} \Delta U\right) /\left(\alpha_{\mathrm{p}} \rho_{\mathrm{f}}+\alpha_{\mathrm{f}} \rho_{\mathrm{p}}\right)$ due to the sudden increment $\Delta U$ at $t=t_{0}$. This is not the same as LEM. As $\left(\alpha_{\mathrm{f}}\right)_{t_{0+}}=\left(\alpha_{\mathrm{f}}\right)_{t_{0-}},\left(u_{\mathrm{f}}-\right.$ $\left.u_{\mathrm{p}}\right)_{t_{0+}}=\left(u_{\mathrm{f}}-u_{\mathrm{p}}\right)_{t_{0-}}+\left(\Delta u_{\mathrm{f}}\right)_{\mathrm{TFM}}-\left(\Delta u_{\mathrm{p}}\right)_{\mathrm{TFM}}=\left(u_{\mathrm{f}}-u_{\mathrm{p}}\right)_{\mathrm{t}_{0-}}+\left(\rho_{\mathrm{p}}-\right.$ $\left.\rho_{\mathrm{f}}\right) \Delta U /\left(\alpha_{\mathrm{p}} \rho_{\mathrm{f}}+\alpha_{\mathrm{f}} \rho_{\mathrm{p}}\right) \neq u_{\mathrm{T}}\left(\alpha_{\mathrm{f}}\right)_{t_{0+}} \mathrm{n}-1$, there is a relaxation process for the relative velocity between the two phases to adjust itself into another equilibrium state.

For the simple bed expansion and bed collapse processes, the fluidizing velocity $U$ does not change after $t=t_{0+}$, and the distribution of various parameters can be assumed to be uniform in the bed before the ending of the relaxation process. ${ }^{5}$ Under such conditions, the solid phase velocity and the relative velocity between the two phases in the relaxation process are (see Appendix)

$$
\begin{aligned}
u_{\mathrm{p}}(t)= & {\left[U\left(t_{0+}\right)-u_{\mathrm{T}} \alpha_{\mathrm{f}}^{n}\right]\left[1-\exp \left(-\left(t-t_{0+}\right) / \tau_{\mathrm{sys}}\right)\right] } \\
& +u_{\mathrm{p}}\left(t_{0+}\right) \exp \left(-\left(t-t_{0+}\right) / \tau_{\text {sys }}\right) \\
u_{\mathrm{f}}(t)-u_{\mathrm{p}}(t)= & \left.\left(u_{\mathrm{f}}-u_{\mathrm{p}}\right)\right|_{t_{0-}}\left[1-\exp \left(-\left(t-t_{0+}\right) / \tau_{\text {sys }}\right)\right] \\
& +\left.\left(u_{\mathrm{f}}-u_{\mathrm{p}}\right)\right|_{t_{0+}} \exp \left(-\left(t-t_{0+}\right) / \tau_{\text {sys }}\right)
\end{aligned}
$$

where the relaxation time of the two-phase system $\tau_{\text {sys }}$ is

$$
\tau_{\text {sys }}=\frac{u_{\mathrm{T}} \alpha_{\mathrm{f}}^{n}}{\left(1-\rho_{\mathrm{f}} / \rho_{\mathrm{p}}\right) g}\left[\frac{\alpha_{\mathrm{f}} \rho_{\mathrm{p}}+\alpha_{\mathrm{p}} \rho_{\mathrm{f}}}{\alpha_{\mathrm{f}} \rho_{\mathrm{p}}}\right]=\tau_{\mathrm{p}} \alpha_{\mathrm{f}}^{n}\left[1+\frac{\alpha_{\mathrm{p}}}{\alpha_{\mathrm{f}}} \frac{\rho_{\mathrm{f}}}{\rho_{\mathrm{p}}}\right] \text {, }
$$

and $\tau_{\mathrm{p}}=\frac{u_{\mathrm{T}}}{\left(1-\rho_{\mathrm{f}} / \rho_{\mathrm{p}}\right) g}$ is the relaxation time for a single particle. For the particle-liquid system in this study, $\tau_{\mathrm{p}} \approx 0.032 \mathrm{~s}$.

In the expansion process when the fluidizing velocity $U$ suddenly increases from $U=U_{1}=0.055 \mathrm{~m} / \mathrm{s}$ to $U=U_{2}=$ $0.128 \mathrm{~m} / \mathrm{s}, \quad \tau_{\text {sys }} \approx 0.36 \tau_{\mathrm{p}} \approx 0.012 \mathrm{~s}$, and in the collapse process when $U$ decreases from $U=U_{2}=0.128 \mathrm{~m} / \mathrm{s}$ to $U=U_{1}=0.055 \mathrm{~m} / \mathrm{s}, \tau_{\text {sys }} \approx 0.81 \tau_{\mathrm{p}} \approx 0.026 \mathrm{~s}$. The two relaxation processes after the sudden changes of $U$ are shown in

\footnotetext{
${ }^{5}$ Assuming that the relaxation process nearly ends at $t=t_{0}+\delta t$, then the non-uniform section is only limited in a layer no more than $l(l=V \cdot \delta t)$ above the distributor, where $V$ is the velocity of concentration wave. From the discussion below, $\delta t$ is usually very short (only several times of the system relaxation time), so the length scale of non-uniform region is very small.
} 

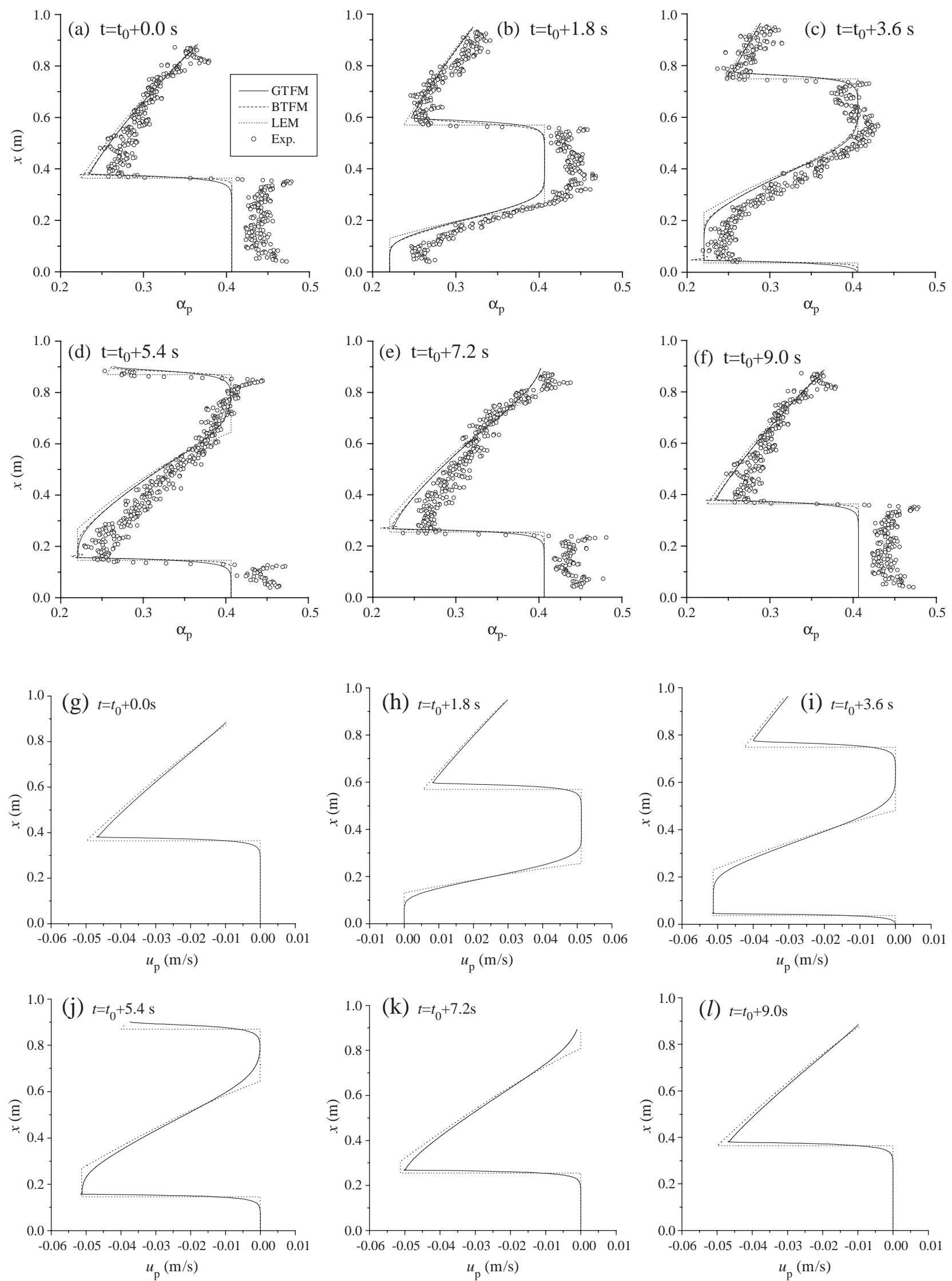

Fig. 9. Distribution of particle concentration $(\mathrm{a}-\mathrm{f})$ and particle velocity $(\mathrm{g}-1)$ at different times in a period $\left(s=0.1, C_{\mu}=0, C_{\mathrm{vm}}=0.5\right.$ in $\left.\mathrm{GTFM}\right)$. 

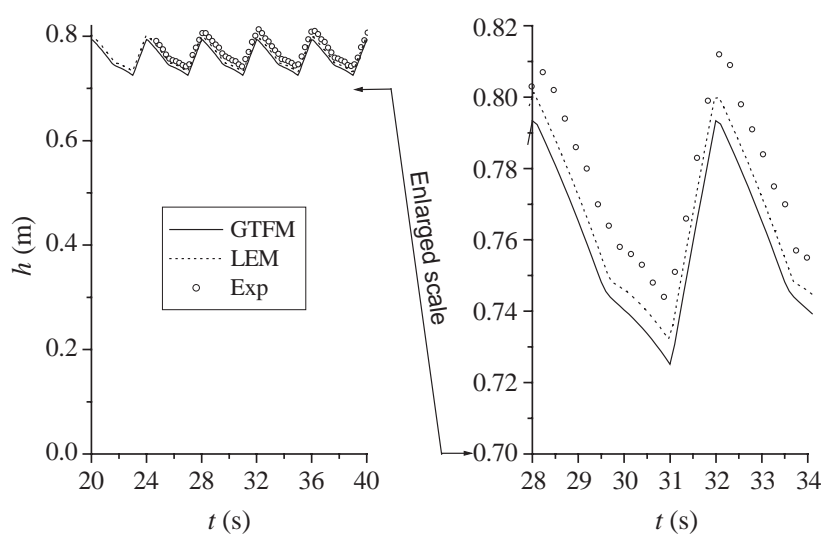

Fig. 10. Bed height variations with time responding to a periodically pulsed fluidizing velocity.

Fig. 12. In order to be convenient for comparison, the changes of velocity from LEM are also shown in the figure.

From Fig. 12, Eqs. (31) and (32), when $\left(t-t_{0+}\right)$ passes several times of $\tau_{\text {sys }}$ (about $m \approx 3 \sim 4$ times), $\left.u_{\mathrm{p}}(t)\right|_{t>t_{0}+m \tau_{\mathrm{sys}}} \approx U\left(t_{0+}\right)-u_{\mathrm{T}} \alpha_{\mathrm{f}}^{n}=\Delta U+u_{\mathrm{p}}\left(t_{0-}\right)=\left.\left(u_{\mathrm{p}}\right)_{\mathrm{LEM}}\right|_{t>t_{0+}}$, $\left.\left[u_{\mathrm{f}}(t)-u_{\mathrm{p}}(t)\right]_{t>t_{0}+m \tau_{\mathrm{sys}}} \approx\left(u_{\mathrm{f}}-u_{\mathrm{p}}\right)\right|_{t_{0-}}=\left.\left(u_{\mathrm{f}}-u_{\mathrm{p}}\right)_{\mathrm{LEM}}\right|_{t>t_{0+}}$, that is, the velocities of two phases almost reach the equilibrium values computed from LEM.

If the virtual mass force $F_{\mathrm{vm}}$ expressed by Eq. (11) is included in the inter-phase force $F_{\mathrm{p}}$, then the relaxation time of the system is (see Appendix)

$$
\begin{aligned}
\tau_{\text {sys }}= & \frac{u_{\mathrm{T}} \alpha_{\mathrm{f}}^{n}}{\left(\rho_{\mathrm{p}}-\rho_{\mathrm{f}}\right) g}\left(\left(\rho_{\mathrm{p}}+C_{\mathrm{vm}} \rho_{\mathrm{f}} / \alpha_{\mathrm{f}}\right)\right. \\
& \left.+\left(\alpha_{\mathrm{p}} / \alpha_{\mathrm{f}}\right)\left(\rho_{\mathrm{f}}-C_{\mathrm{vm}} \rho_{\mathrm{f}} / \alpha_{\mathrm{f}}\right)\right) .
\end{aligned}
$$

The above analyses show that ignoring the difference of inertial forces between the two phases introduces certain errors in a very short period only about tens of milliseconds after $U$ changes suddenly. After the period, LEM is applicable.

The errors caused by ignoring the difference of inertia forces between the two phases in a non-uniform flow field are analyzed hereinafter. For example, the non-uniform flow field exists in the region $t V\left(\alpha_{\mathrm{f}, 2}, U_{2}\right)<x<t V\left(\alpha_{\mathrm{f}, 1}, U_{2}\right)$ in simple expansion process. In this region, using Eqs. (1), (2'), (5) and (27), the ratio of the difference of the inertial forces and gravity force corrected by buoyancy can be written as

$$
\begin{aligned}
& \frac{1}{\left(\rho_{\mathrm{p}}-\rho_{\mathrm{f}}\right) g}\left[\rho_{\mathrm{p}}\left(\frac{\partial u_{\mathrm{p}}}{\partial t}+u_{\mathrm{p}} \frac{\partial u_{\mathrm{p}}}{\partial x}\right)-\rho_{\mathrm{f}}\left(\frac{\partial u_{\mathrm{f}}}{\partial t}+u_{\mathrm{f}} \frac{\partial u_{\mathrm{f}}}{\partial x}\right)\right] \\
& =\frac{\left(u_{\mathrm{T}} n \alpha_{\mathrm{f}}^{n-1}\right)^{2}}{\left(\rho_{\mathrm{p}}-\rho_{\mathrm{f}}\right) g} \frac{\partial \alpha_{\mathrm{f}}}{\partial x}\left[\alpha_{\mathrm{p}} \rho_{\mathrm{p}}+\left(\frac{n-1}{n \alpha_{\mathrm{f}}}-1\right)^{2} \alpha_{\mathrm{f}} \rho_{\mathrm{f}}\right] \\
& =-\Phi \frac{\tau_{\mathrm{p}}}{t}
\end{aligned}
$$

where

$$
\begin{aligned}
\Phi=\Phi\left(n, \alpha_{\mathrm{f}}, \frac{\rho_{\mathrm{p}}}{\rho_{\mathrm{f}}}\right)= & {\left[1+\frac{\rho_{\mathrm{f}}}{\alpha_{\mathrm{f}} \alpha_{\mathrm{p}} \rho_{\mathrm{p}}}\left(\frac{n-1}{n}-\alpha_{\mathrm{f}}\right)^{2}\right] } \\
& \times \frac{n \alpha_{\mathrm{f}}^{n} \alpha_{\mathrm{p}}}{\left[(n+1) \alpha_{\mathrm{f}}-(n-1)\right]} .
\end{aligned}
$$

$\Phi \approx 0.44$ when $n=2.414, \rho_{\mathrm{p}} / \rho_{\mathrm{f}}=2.6, \alpha_{\mathrm{f}}=0.6, \Phi$ continues to decrease with the increase of $\alpha_{\mathrm{f}}$. Therefore, the errors caused by ignoring the difference of inertia forces between the two phases become very small when $t$ passes several times of $\tau_{\mathrm{p}}$.

\subsection{Structure of the particle concentration discontinuity in simple collapse process}

Eqs. (1), (2'), (5), (27) show that the flow field is continuous except for finite number of discontinuities in the pulsed flow field. According to LEM, these discontinuities are surfaces without any thickness. The relative velocity between the two phases has a jump change through the discontinuity surfaces. The difference of the inertial forces between the two phases thus must have an important influence where the relative velocity has a jump. Therefore, it is improper to ignore the difference of inertia near the discontinuity surfaces. In experiment, one can observe that the discontinuity has certain thickness, though it is very thin. The transition from the upper dilute section to the lower dense section in the collapse process is analyzed using GTFM.

According to the experimental observation, the discontinuity speed $V_{S}$ does not change during the collapse process (it can also be derived from LEM, Eq. (30)). First, we make a coordinates transform using a moving coordinate $\xi$ with the discontinuity speed $V_{s}$ to substitute the laboratory coordinate $x$, i.e., $\xi=x-V_{s} t$. Then, the flow is steady in the moving coordinate, i.e., $\left.\frac{\partial \varphi}{\partial t}\right|_{\xi}=0$. For any variable $\varphi$, there are $\left.\frac{\partial \varphi}{\partial t}\right|_{x}=\left.\frac{\partial \varphi}{\partial t}\right|_{\xi}-\left.V_{s} \frac{\partial \varphi}{\partial \xi}\right|_{t}=-\left.V_{s} \frac{\partial \varphi}{\partial \xi}\right|_{t}=-\left.\frac{\partial\left(\varphi V_{s}\right)}{\partial \xi}\right|_{t}$, $\left.\frac{\partial \varphi}{\partial x}\right|_{t}=\left.\frac{\partial \varphi}{\partial \xi}\right|_{t}$. Eqs. (1) and (3') can be transformed into

$$
\begin{aligned}
& \frac{d\left[\alpha_{\mathrm{p}}\left(u_{\mathrm{p}}-V_{s}\right)\right]}{d \xi}=0, \\
& \rho_{\mathrm{p}}\left(u_{\mathrm{p}}-V_{s}\right) \frac{d u_{\mathrm{p}}}{d \xi}-\rho_{\mathrm{f}}\left(u_{\mathrm{f}}-V_{s}\right) \frac{d u_{\mathrm{f}}}{d \xi} \\
& \quad=\frac{F_{\mathrm{p}}}{\alpha_{\mathrm{p}} \alpha_{\mathrm{f}}}-\left(\rho_{\mathrm{p}}-\rho_{\mathrm{f}}\right) g+\frac{1}{\alpha_{\mathrm{p}}} \frac{d\left(-p_{\mathrm{p}}+\tau_{\mathrm{p}, \mathrm{xx}}\right)}{d \xi} .
\end{aligned}
$$

Eqs. (8), (11), (14) and (16) are used to model the momentum exchange coefficient $\beta$, the virtual mass force $F_{\text {vm }}$, the solid phase pressure $p_{\mathrm{p}}$ and the viscous coefficient $\mu_{\mathrm{p}}$, respectively, where $s, C_{\mathrm{vm}}$ and $C_{\mu}$ are model parameters. Letting $\rho_{\mathrm{r}}=\rho_{\mathrm{f}} / \rho_{\mathrm{f}}, \quad L_{\mathrm{a}}=\alpha_{\mathrm{p}, 1} \alpha_{\mathrm{p}, 2}\left(\alpha_{\mathrm{f}, 2}^{n}-\alpha_{\mathrm{f}, 1}^{n}\right) /\left(\alpha_{\mathrm{p}, 1}-\alpha_{\mathrm{p}, 2}\right)$, $L_{\mathrm{b}}=\alpha_{\mathrm{f}, 1} \alpha_{\mathrm{f}, 2}\left(\alpha_{\mathrm{p}, 1} \alpha_{\mathrm{f}, 1}^{n-1}-\alpha_{\mathrm{p}, 2} \alpha_{\mathrm{f}, 2}^{n-1}\right) /\left(\alpha_{\mathrm{p}, 1}-\alpha_{\mathrm{p}, 2}\right), L_{\mathrm{c}}=\alpha_{\mathrm{p}, \mathrm{c}} /$ $\left(\alpha_{\mathrm{p}, \mathrm{c}}-\alpha_{\mathrm{p}}\right)^{2}, \quad L_{\mathrm{d}}=(3 / 4) C_{\mu}\left(1-\rho_{\mathrm{r}}\right) g d_{\mathrm{p}} / u_{\mathrm{T}}^{2}, \quad Y=\xi\left(1-\rho_{\mathrm{r}}\right) g /$ $u_{\mathrm{T}}^{2}, B=s L_{\mathrm{a}} / \alpha_{\mathrm{p}}-\left(1+C_{\mathrm{vm}} \rho_{\mathrm{r}} / \alpha_{\mathrm{f}}\right) L_{\mathrm{a}}^{2} / \alpha_{\mathrm{p}}^{3}-\rho_{\mathrm{r}}\left(1+C_{\mathrm{vm}} / \alpha_{\mathrm{f}}\right) L_{\mathrm{b}}^{2} /$ $\alpha_{\mathrm{f}}^{3}, Q=1-\left[\alpha_{\mathrm{p}, 1} \alpha_{\mathrm{f}, 1}^{n} /\left(\alpha_{\mathrm{p}, 1}-\alpha_{\mathrm{p}, 2}\right)-\alpha_{\mathrm{p}, 2} \alpha_{\mathrm{f}, 2}^{n} /\left(\alpha_{\mathrm{p}, 1}-\alpha_{\mathrm{p}, 2}\right)+L_{\mathrm{a}} /\right.$ $\left.\alpha_{\mathrm{p}}\right] \alpha_{\mathrm{f}}^{-n}$, one can get $u_{\mathrm{p}}=V_{s}-L_{\mathrm{a}} u_{\mathrm{T}} / \alpha_{\mathrm{p}}$ and $u_{\mathrm{f}}=V_{s}+L_{\mathrm{b}} u_{\mathrm{T}} / \alpha_{\mathrm{f}}$ from Eqs. (37) and (2'). Introducing them into Eq. (38) yields

$$
\begin{aligned}
B\left(\frac{d Y}{d \alpha_{\mathrm{p}}}\right)^{-1}= & -Q-L_{\mathrm{d}} L_{\mathrm{a}}\left[\frac{1}{\left(\alpha_{\mathrm{p}, \mathrm{c}}-\alpha_{\mathrm{p}}\right) \alpha_{\mathrm{p}}^{3}}\left(\frac{d Y}{d \alpha_{\mathrm{p}}}\right)^{-3} \frac{d^{2} Y}{d \alpha_{\mathrm{p}}^{2}}\right. \\
& \left.+\frac{2 \alpha_{\mathrm{p}, \mathrm{c}}-3 \alpha_{\mathrm{p}}}{\left(\alpha_{\mathrm{p}, \mathrm{c}}-\alpha_{\mathrm{p}}\right)^{2} \alpha_{p}^{4}}\left(\frac{d Y}{d \alpha_{\mathrm{p}}}\right)^{-2}\right]
\end{aligned}
$$


For a small value of $C_{\mu}$, the above equation can be approximated as

$$
\begin{aligned}
\frac{d Y}{d \alpha_{\mathrm{p}}}= & -\frac{B}{Q}\left\{1-L_{\mathrm{d}} L_{\mathrm{a}}\left[\frac{1}{\left(\alpha_{\mathrm{p}, \mathrm{c}}-\alpha_{\mathrm{p}}\right) \alpha_{\mathrm{p}}^{3}} \frac{B^{\prime} Q-B Q^{\prime}}{B^{3}}\right.\right. \\
& \left.\left.+\frac{2 \alpha_{\mathrm{p}, \mathrm{c}}-3 \alpha_{\mathrm{p}}}{\left(\alpha_{\mathrm{p}, \mathrm{c}}-\alpha_{\mathrm{p}}\right)^{2} \alpha_{\mathrm{p}}^{4}} \frac{Q}{B^{2}}\right]\right\} .
\end{aligned}
$$

where $B^{\prime}$ and $Q^{\prime}$ are the derivatives of $B$ and $Q$ to $\alpha_{\mathrm{p}}$, respectively.

The asymptotic conditions are: $Y \rightarrow+\infty$ when $\alpha_{\mathrm{p}} \rightarrow \alpha_{\mathrm{p}, 2}$, and $Y \rightarrow-\infty$ when $\alpha_{\mathrm{p}} \rightarrow \alpha_{\mathrm{p}, 1}$. Integrating Eq. (39) from $\alpha_{\mathrm{p}, 2}$ to $\alpha_{\mathrm{p}, 1}$, the line for $\alpha_{\mathrm{p}}(Y)$ is shown in Fig. 13. For comparison, the result about $\alpha_{\mathrm{p}}(Y)$ computed from LEM is also shown in the figure (represented by a solid line, there is a discontinuity at $Y=0)$.

From the above results, one can see that the particle concentration gradually transits from the upper dilute section to the lower dense section when the inertial force, the solid phase pressure, the viscous stress, and the virtual mass forces are considered in GTFM. The thickness of the transition layer depends on the values of the coefficients $s$,
$C_{\mathrm{vm}}$ and $C_{\mu}$. Letting the three coefficients be zero and only maintaining the inertia terms of the two phases, one cannot get the reasonable solution from BTFM. Therefore, the three forces are very important. Further researches show that the most important term is the solid phase pressure and the variation of the coefficient $s$ greatly influences the thickness of the transition layer (Fig. 13a). When the coefficient $s$ is very small (its exact value depends on $C_{\mathrm{vm}}$ and $C_{\mu}$, generally speaking, when it is less than $0.1 \sim 0.2$ ), the correct solution does not exist. $C_{\mathrm{vm}}$ and $C_{\mu}$ may be zero (Fig. 13(b) and (c)), but $s$ cannot be zero.

The above results are only of qualitative significance, because the models used in this study for the virtual mass force $F_{\mathrm{vm}}$, the solid phase pressure $p_{\mathrm{p}}$ and the viscous coefficient $\mu_{\mathrm{p}}$ cannot be accurately determined and the values are chosen at will to some extent.

It is not true that the thickness of transition layer is zero and there is a jump for every parameter through the discontinuity surface according to LEM. In fact, the transition layer has certain thickness and every parameter transits smoothly (but quickly). However, this layer is very thin (commonly it is only several millimeters, equivalent to several times of particle diameter), LEM is approximately
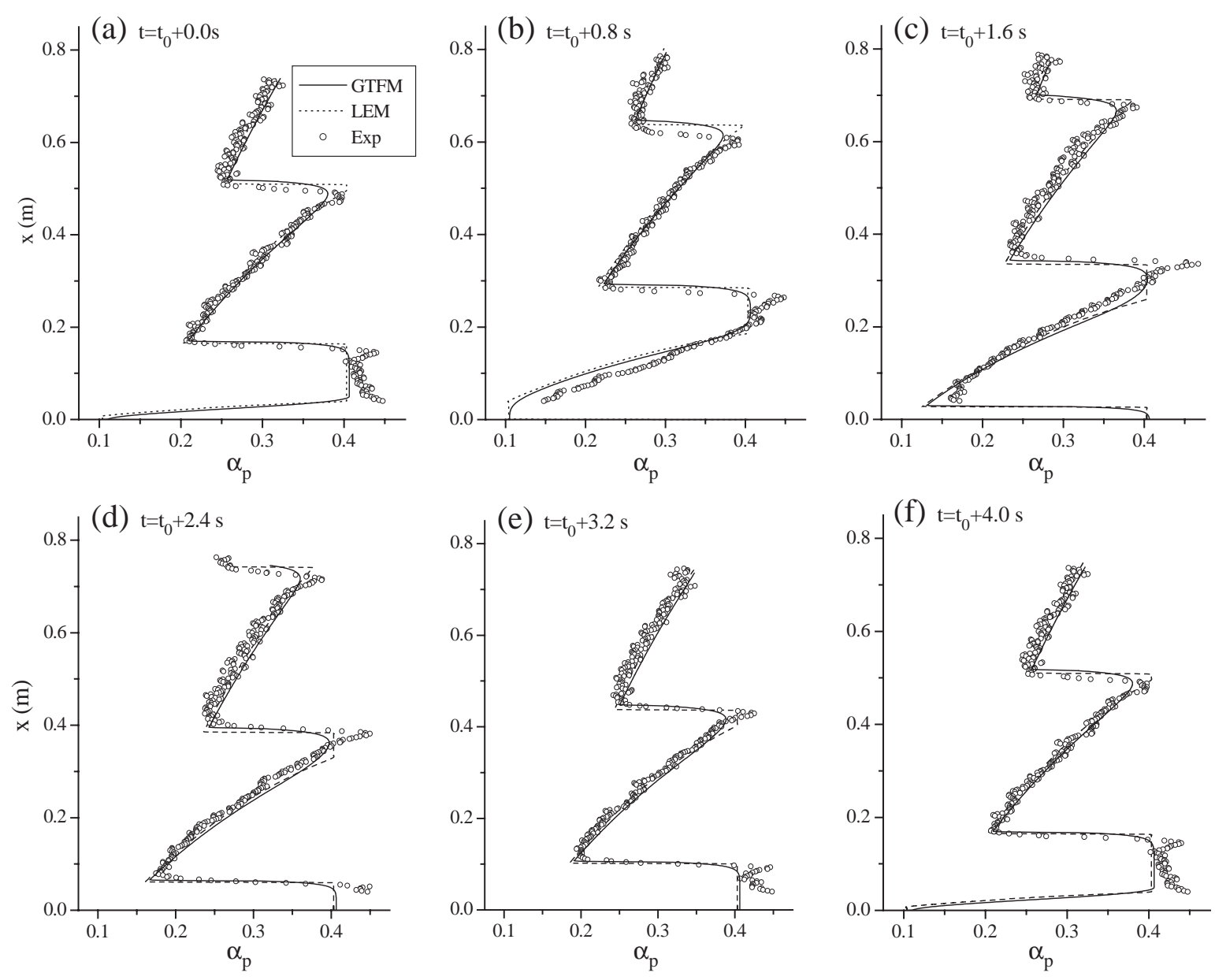

Fig. 11. Distribution of particle concentration $(\mathrm{a}-\mathrm{f})$ and particle velocity $(\mathrm{g}-1)$ at different times in a period $\left(s=0.07, C_{\mu}=0, C_{\mathrm{vm}}=0.5\right.$ in GTFM). 

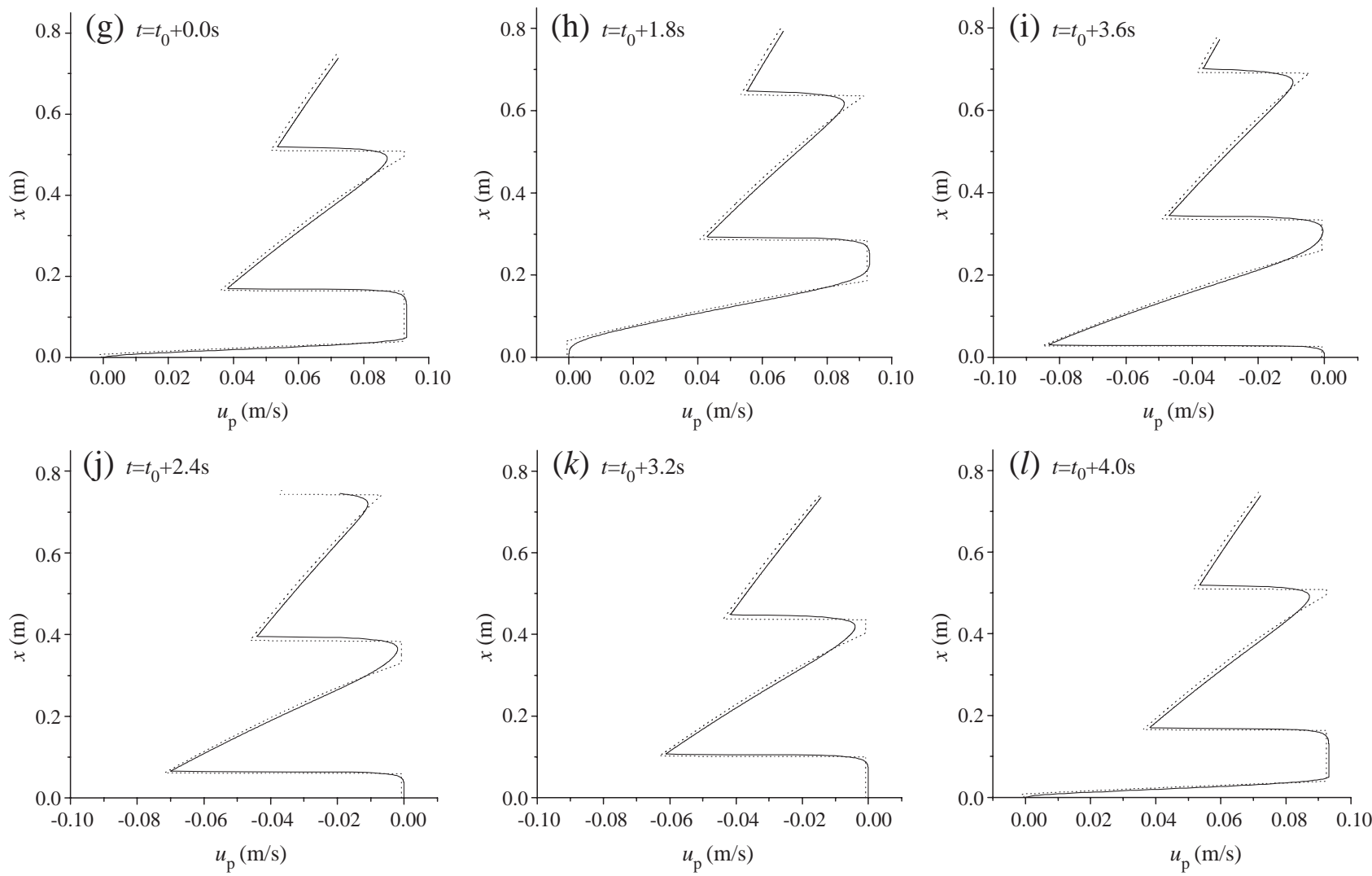

Fig. 11 (continued).

proper except for the sections near the discontinuity surfaces.

In order to get reasonable results from GTFM, the principle for choosing the coefficients $s, C_{\mathrm{vm}}$ and $C_{\mu}$ is to insure the eigenvalues be real numbers firstly, then to let them be as small as possible because the too thick transitional layer does not fit the result observed in the experiment.

In conclusion, the errors in the simulation results from LEM only exist in a time interval of tens of milliseconds (several times of the particle relaxation time) and a spatial

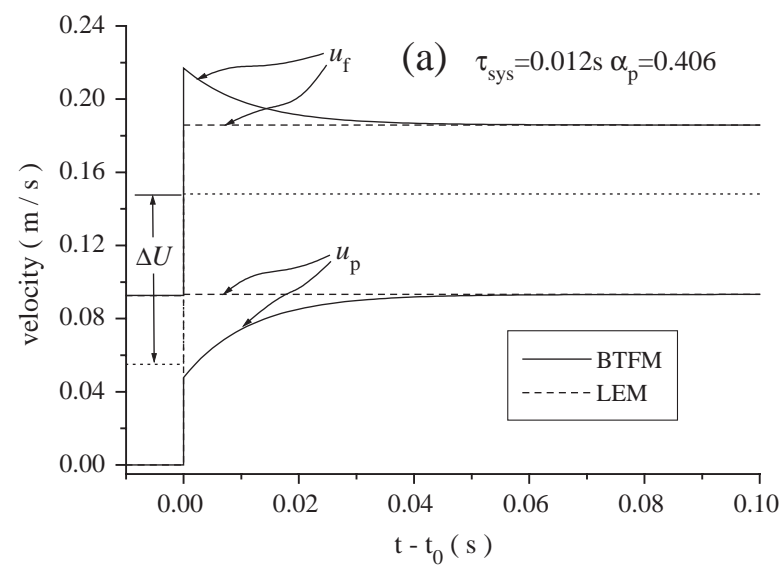

interval of several millimeters (several times of the particle diameter) when simulating the pulsed flow in the fluidized bed. The above limitations of LEM are insignificant for practical simulation to capture the main characteristics of the pulsed two-phase flow.

\section{Discussions and conclusions}

1) It is visually observed that concentration waves and shock waves continuously travel upwards from the

Fig. 12. The relaxation processes of the solid and fluid phase velocity when $U$ suddenly changes. 

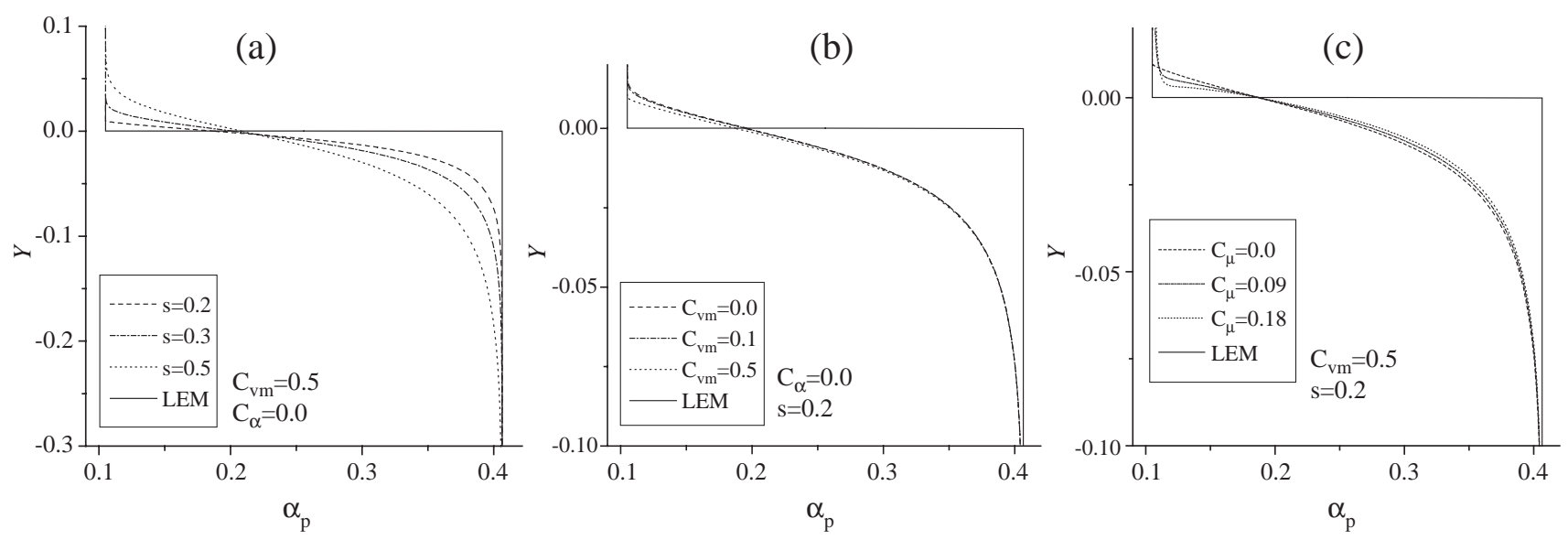

Fig. 13. Influence of $s, C_{\mathrm{vm}}$ and $C_{\mu}$ on the structure of the transitional layer.

distributor as the pattern of a planar wave, resulting from the periodical variation of fluidizing velocity $U(t)$ (see Fig. 1), which demonstrates that the pulsating twophase flow in the bed is almost one-dimensional. The good agreement between the numerical results and experimental data further accounts for the rationality of the one-dimensional model for the flow studied in this paper.

2) Although GTFM is a general model, in which various influencing factors such as the solid phase pressure $p_{\mathrm{p}}$, the viscous stress $\tau_{\mathrm{p}, \mathrm{xx}}$ and the virtual mass force $F_{\mathrm{vm}}$ are included, researches on the constitutive relationships of $p_{\mathrm{p}}, \tau_{\mathrm{p}, \mathrm{xx}}$ and $F_{\mathrm{vm}}$ for closing the model yet are not satisfying, the expressions used in different references are distinct from each other. For a given choosing of constitutive relationships of $p_{\mathrm{p}}, \tau_{\mathrm{p}, \mathrm{xx}}$ and $F_{\mathrm{vm}}$, the result of the flow in pulsed bed can be gotten by numerically solving GTFM, however, these results imply great undeterminedness due to the adjustable coefficients in these constitutive relationships.

3 ) It is thought that the values of $p_{\mathrm{p}}, \tau_{\mathrm{p}, \mathrm{xx}}$ and $F_{\mathrm{vm}}$ are small compared with those of the inter-phase drag force and gravity force corrected by buoyancy. Many researches have been based on BTFM, in which the terms of $p_{\mathrm{p}}$, $\tau_{\mathrm{p}, \mathrm{xx}}$ and $F_{\mathrm{vm}}$ are completely neglected. BTFM hardly has adjustable parameters, thus reducing the undeterminedness of the numerical results. However, the eigenvalues of BTFM contains complex numbers, i.e., the model is ill-posed for initial-value problems. The numerical simulation using this model often fails with grid refinement because the model is inherently unstable for small disturbances.

When the amplitude of fluidizing velocity $\Delta U$ is small, the imaginary part of the eigenvalues is also small, the numerical viscosity or artificial viscosity can be used to suppress the instability (see Case A in Figs. 8 and 9). However, when the amplitude of fluidizing velocity $\Delta U$ is large, the imaginary part of the eigenvalues becomes large. It is difficult to suppress the numerical instability using the numerical viscosity or artificial viscosity (see Case B in Figs. 10 and 11).

4) Inertial terms are related to the ill-posedness of BTFM. LEM can be obtained by neglecting the difference of the inertial forces between the two phases based on BTFM. From BTFM to LEM, the equation set reduces into a first order partial differential equation, the two complex eigenvalue degenerates into a real number, the difficulty in numerical simulation resulting from the illposedness is thus overcome. LEM contains neither the undeterminedness due to the adjustable parameters in GTFM nor the ill-posedness of BTFM. The good agreement between the numerical results and experimental data shows that LEM has acceptable accuracy as a whole for studying the flow in liquid pulsed fluidized bed, and it is also demonstrated that, as a first approximation, it is reasonable to neglect $p_{\mathrm{p}}, \tau_{\mathrm{p}, \mathrm{xx}}, F_{\mathrm{vm}}$ and the difference of the inertial forces between two phases because they are small as compared with the inter-phase drag force and the gravity corrected by buoyancy in most time of the unsteady flow and most places in the non-uniform flow field.

5) Just as the detailed information in the wall boundary layer of the single-phase flow is lost when the NavierStokes equation set is simplified into the Euler equation set, LEM loses the capability to capture some details of the two-phase flow in pulsed fluidized bed.

The velocities of the two phases get different increments because of the different inertia when fluidizing velocity has a jump change. The aftereffect caused by the different inertia vanishes after a short relaxation process following the jump change. In LEM, the different increments for the velocities and the relaxation process are lost because the difference of the inertial forces is neglected.

The concentration shock obtained from LEM is a geometrical surface without any thickness for the same reason that the difference of the inertial forces is neglected. In fact, the very large gradients of concen- 
tration and velocities are finite in strength near the discontinuity surfaces in the flow field, the difference of the inertial forces is also very important, thus making the concentration shocks have certain thickness. The flow structure in concentration shock is lost using LEM.

The relaxation process after the jump change of fluidizing velocity is very short (only tens of milliseconds, i.e., several times of system relaxation time) and the real thickness of the concentration shocks is very thin (only several millimeters, i.e., several times of particle diameter), the above limitations of LEM do not hinder it from capturing the main characteristics of the pulsed flow.

6) The model developed and conclusions drawn in this paper are restricted in the area of liquid pulsed fluidization. Because of the difference in mechanisms of inter-particle interactions and the dynamic response of phases between the gas and liquid fluidization, the study in this paper just establish a basis for further studying the gas fluidization, and one should be careful to extend the model and the resulted conclusions for liquid fluidization directly to gas fluidization without capturing all the major mechanism difference and performing experimental validation. The effects of inertia difference between gas and solid phases and particle collisions on the applicability of LEM to gas fluidization deserve further analysis and experimental validation.

\section{Acknowledgments}

The authors acknowledge the support from NSFC grant 20376083 and the Multiphase Reaction Laboratory, Institute of Process Engineering, Chinese Academy of Sciences. The authors are also grateful for the help from Dr. Jijia Xie, who helped to transform the gray scale of photos into data.

\section{Appendix A}

In a uniform fluidized bed $(\partial / \partial x=0)$, from Eq. (1), one can know,

$\Delta \alpha_{\mathrm{p}}=\int_{t_{0-}}^{t_{0+}} \frac{\partial \alpha_{\mathrm{p}}}{\partial t} d t=0 \quad$ and $\left(\alpha_{\mathrm{p}}\right)_{t_{0+}}=\left(\alpha_{\mathrm{p}}\right)_{t_{0-}}=\alpha_{\mathrm{p}}$.

Before and after the sudden change of fluidizing velocity, the continuity equations for the mixture are:

$$
\begin{aligned}
& \alpha_{\mathrm{p}} u_{\mathrm{p}, t_{0-}}+\left(1-\alpha_{\mathrm{p}}\right) u_{\mathrm{f}, t_{0-}}=U_{t_{0-}} \\
& \alpha_{\mathrm{p}} u_{\mathrm{p}, t_{0+}}+\left(1-\alpha_{\mathrm{p}}\right) u_{\mathrm{f}, t_{0+}}=U_{t_{0+}}
\end{aligned}
$$

Subtracting Eq. (A-2) from Eq. (A-3), one can get

$\alpha_{\mathrm{p}} \Delta u_{\mathrm{p}}+\left(1-\alpha_{\mathrm{p}}\right) \Delta u_{\mathrm{f}}=\Delta U$

where $\Delta u_{\mathrm{p}}=u_{\mathrm{p}, t_{0+}}-u_{\mathrm{p}, t_{0-}}, \quad \Delta u_{\mathrm{f}}=u_{\mathrm{f}, \boldsymbol{t}_{0+}}-u_{\mathrm{f}, t_{0-}}$ and $\Delta U=U_{t_{0+}}-U_{t_{0-}}$.

Using Eq. (8), Eq. ( $\left.3^{\prime}\right)$ in the uniform fluidized can be expressed as,

$$
\begin{aligned}
& \left(\rho_{\mathrm{p}}+C_{\mathrm{vm}} \rho_{\mathrm{f}} / \alpha_{\mathrm{f}}\right) \frac{\partial u_{\mathrm{p}}}{\partial t}-\left(\rho_{\mathrm{f}}-C_{\mathrm{vm}} \rho_{\mathrm{f}} / \alpha_{\mathrm{f}}\right) \frac{\partial u_{\mathrm{f}}}{\partial t} \\
& =\left(\rho_{\mathrm{f}}-\rho_{\mathrm{p}}\right) g\left(\frac{u_{\mathrm{f}}-u_{\mathrm{p}}}{u_{\mathrm{T}} \alpha_{\mathrm{f}}^{n-1}}-1\right) .
\end{aligned}
$$

Integrating both sides of Eq. (A-5) from $t_{0-}$ to $t_{0+}$ yields,

$$
\begin{aligned}
\left(\rho_{\mathrm{p}}\right. & \left.+C_{\mathrm{vm}} \rho_{\mathrm{f}} / \alpha_{\mathrm{f}}\right) \Delta u_{\mathrm{p}}-\left(\rho_{\mathrm{f}}-C_{\mathrm{vm}} \rho_{\mathrm{f}} / \alpha_{\mathrm{f}}\right) \Delta u_{\mathrm{f}} \\
= & \int_{t_{0-}}^{t_{0+}}\left(\rho_{\mathrm{p}} \frac{\partial u_{\mathrm{p}}}{\partial t}-\rho_{\mathrm{f}} \frac{\partial u_{\mathrm{f}}}{\partial t}\right) d t \\
= & \int_{t_{0-}}^{t_{0+}}\left(\rho_{\mathrm{f}}-\rho_{\mathrm{p}}\right) g\left(\frac{u_{\mathrm{f}}-u_{\mathrm{p}}}{u_{\mathrm{T}} \alpha_{\mathrm{f}}^{n-1}}-1\right) d t=0 .
\end{aligned}
$$

From (A-4) and (A-6), one can get the increments of solid phase and liquid phase respectively,

$$
\begin{aligned}
\left(\Delta u_{\mathrm{p}}\right)_{\mathrm{TFM}}= & \left(\rho_{\mathrm{f}}-C_{\mathrm{vm}} \rho_{\mathrm{f}} / \alpha_{\mathrm{f}}\right) \Delta U /\left(\alpha_{\mathrm{p}}\left(\rho_{\mathrm{f}}-C_{\mathrm{vm}} \rho_{\mathrm{f}} / \alpha_{\mathrm{f}}\right)\right. \\
& \left.+\alpha_{\mathrm{f}}\left(\rho_{\mathrm{p}}+C_{\mathrm{vm}} \rho_{\mathrm{f}} / \alpha_{\mathrm{f}}\right)\right), \\
\left(\Delta u_{\mathrm{f}}\right)_{\mathrm{TFM}}= & \left(\rho_{\mathrm{p}}+C_{\mathrm{vm}} \rho_{\mathrm{f}} / \alpha_{\mathrm{f}}\right) \Delta U /\left(\alpha_{\mathrm{p}}\left(\rho_{\mathrm{f}}-C_{\mathrm{vm}} \rho_{\mathrm{f}} / \alpha_{\mathrm{f}}\right)\right. \\
& \left.+\alpha_{\mathrm{f}}\left(\rho_{\mathrm{p}}+C_{\mathrm{vm}} \rho_{\mathrm{f}} / \alpha_{\mathrm{f}}\right)\right) .
\end{aligned}
$$

From Eq. (2'), one can get,

$u_{\mathrm{f}}=\left(U-\alpha_{\mathrm{p}} u_{\mathrm{p}}\right) /\left(1-\alpha_{\mathrm{p}}\right)$.

Introducing (A-7) into (A-5), one can obtain,

$$
\begin{aligned}
\frac{\partial u_{\mathrm{p}}}{\partial t}= & \frac{U-u_{\mathrm{p}}}{\tau_{\text {sys }}} \\
& -\frac{\left(\rho_{\mathrm{p}}-\rho_{\mathrm{f}}\right) g}{\rho_{\mathrm{p}}+C_{\mathrm{vm}} \rho_{\mathrm{f}} / \alpha_{\mathrm{f}}+\left(\rho_{\mathrm{f}}-C_{\mathrm{vm}} \rho_{\mathrm{f}} / \alpha_{\mathrm{f}}\right)\left(\alpha_{\mathrm{p}} / \alpha_{\mathrm{f}}\right)},
\end{aligned}
$$

where $\tau_{\text {sys }}$ is the relaxation time of the mixture system,

$$
\begin{aligned}
\tau_{\text {sys }}= & \frac{u_{\mathrm{T}} \alpha_{\mathrm{f}}^{n}}{\left(\rho_{\mathrm{p}}-\rho_{\mathrm{f}}\right) g}\left(\left(\rho_{\mathrm{p}}+C_{\mathrm{vm}} \rho_{\mathrm{f}} / \alpha_{\mathrm{f}}\right)+\left(\alpha_{\mathrm{p}} / \alpha_{\mathrm{f}}\right)\right. \\
& \left.\times\left(\rho_{\mathrm{f}}-C_{\mathrm{vm}} \rho_{\mathrm{f}} / \alpha_{\mathrm{f}}\right)\right) .
\end{aligned}
$$

Solving (A-10) yields,

$$
\begin{aligned}
u_{\mathrm{p}}(t)= & {\left[U\left(t_{0+}\right)-u_{\mathrm{T}} \alpha_{\mathrm{f}}^{n}\right]\left[1-\exp \left(-\left(t-t_{0+}\right) / \tau_{\mathrm{sys}}\right)\right] } \\
& +u_{\mathrm{p}}\left(t_{0+}\right) \exp \left(-\left(t-t_{0+}\right) / \tau_{\text {sys }}\right) .
\end{aligned}
$$


Letting $C_{\mathrm{vm}}=0$, Eqs. (A-7), (A-8) and (A-11) degenerate into the equations used in the Subsection 6.1.

\section{References}

[1] D.J. Needham, J.H. Merkin, The propagation of a voidage disturbance in a uniformly fluidized bed, J. Fluid Mech. 13 (1983) 427-454.

[2] M.H.I. Baird, Vibrations and pulsations, Br. Chem. Eng. 11 (1966) $20-25$.

[3] M. Kobayashi, D. Ramaswami, W.T. Brazelton, Heat transfer from an internal surface to a pulsed bed, Chem. Eng. Prog. Symp. Ser. 66 (1970) 58-67.

[4] H.W. Wong, M.H.I. Baird, Fluidization in a pulsed gas flow, Chem. Eng. J. 2 (1971) 104-112.

[5] M. Köksal, H. Vural, Bubble size control in a two-dimensional fluidized bed using a moving double plate distributor, Powder Technol. 95 (1998) 205-213.

[6] A. Nishimura, S. Deguchi, H. Matsuda, M. Hasatani, Solid flow patterns and heat transfer characteristics in a pulsated fluidized bed with flowing large bubbles, Proc. of 11th International Heat Transfer Conference, vol. 4, 1998, pp. 485-490.

[7] D.V. Pence, D.E. Beasley, Heat transfer in pulse-stabilized fluidization: Part 1. Overall cylinder and average local analyses, Int. J. Heat Mass Transfer 45 (2002) 3609-3619.

[8] C.G.J. Baker, T.S.T. Al-Rashidi, A.R. Khan, Studies on liquid-gas and three-phase fluidized beds with pulsating air flows, Can. J. Chem. Eng. 81 (2003) 17-25.

[9] P.L. Slis, T.H.W. Willemse, H. Kramers, The response of the level of a liquid fluidized bed to a sudden change in the fluidizing velocity, Appl. Sci. Res. Section A8 (1959) 209-218.

[10] L.T. Fan, J.A. Schmitz, E.N. Miller, Dynamics of liquid-solid fluidized bed expansion, AIChE J. 9 (1963) 149-153.

[11] L.A.M. van der Wielen, A.W.K.G. Sjauw Koen Fa, J.J.M. Potters, K.Ch.A.M. Luyben, Transient behavior of pulsed particulate fluidized beds, AIChE J. 43 (1997) 625-630.

[12] T.V. Thelen, W.F. Ramirez, Bed-height dynamics of expanded beds, Chem. Eng. Sci. 52 (1997) 3333-3344.

[13] Y.S. Nie, D.Y. Liu, Dynamics of collapsing fluidized beds and its application in the simulation of pulsed fluidized beds, Powder Technol. 99 (1998) 132-139.

[14] T.V. Thelen, W.F. Ramirez, Modeling of solid-liquid fluidization in the Stokes flow regime using two-phase flow theory, AIChE J. 45 (1999) 708-723.

[15] G.D. Jin, Y.S. Nie, D.Y. Liu, Numerical simulation of pulsed fluidization and its experimental validation, Powder Technol. 119 (2001) $153-163$.

[16] S.J. Gelderbloom, D. Gidaspow, R.L. Lyczkowski, CFD simulations of bubbling/collapsing fluidized beds for three Geldart groups, AIChE J. 49 (2003) 844-858.

[17] Q. Zhang, M.P. Zhang, G.D. Jin, D.Y. Liu, C.-W. Shu, Modeling, numerical methods and simulation for particle-fluid two-phase problem, Comput. Math. Appl. 47 (2004) 1437-1462.

[18] Y. Wang, G.S. Gu, F. Wei, W. Jun, Fluidization and agglomerate structure of $\mathrm{SiO}_{2}$ nanoparticle, Powder Technol. 124 (2002) $52-59$.

[19] T.B. Anderson, R. Jackson, A fluid mechanical description of fluidized beds (equations of motion), Ind. Eng. Chem. Fundam. 6 (1967) $527-534$.

[20] D. Gidaspow, Multiphase Flow and Fluidization: Continuum and Kinetic Theory Descriptions, Academic Press, New York, 1994.

[21] H. Enwail, E. Peirano, A.-E. Almstedt, Eulerian two-phase flow theory applied to fluidization, Int. J. Multiph. Flow 22 (1996) 21-66 (Suppl.).
[22] A. Prosperetti, Some considerations on the modeling of disperse multiphase flows by averaged equations, Jsme Int. J. Ser. B Fluids Therm. Eng. 42 (1999) 573-585.

[23] Y.P. Tsuo, D. Gidsspow, Computation of flow patterns in circulating fluidized beds, AIChE J. 36 (1990) 885-896.

[24] I.K. Gamwo, J.S. Halow, D. Gidaspow, R. Mostofi, CFD models for methanol synthesis three-phase reactors: reactor optimization, Chem. Eng. Sci. 93 (2003) 103-112.

[25] A. Kmieć, Particle distributions and dynamics of particle movement in solid-liquid fluidized beds, Chem. Eng. J. 15 (1978) $1-12$.

[26] T. McKeen, T. Pugsley, Simulation and experimental validation of a freely bubbling bed of FCC catalyst, Powder Technol. 129 (2003) $139-152$

[27] K. Agrawal, P. Loezos, M. Syamlal, S. Sundaresan, The role of mesoscale structure in rapid gas-solid flows, J. Fluid Mech. 445 (2001) $151-185$

[28] K.G. Anderson, S. Sundaresan, R. Jackson, Instabilities and the formation of bubbles in fluidized beds, J. Fluid Mech. 303 (1995) 327-366.

[29] P. Duru, M. Nicolas, J. Hinch, É. Guazelli, Constitutive laws in liquidfluidized beds, J. Fluid Mech. 452 (2002) 371-404.

[30] S.C. Tsinontides, R. Jackson, The mechanics of gas fluidized beds with an interval of stable fluidization, J. Fluid Mech. 255 (1993) 237-274.

[31] B.J. Glasser, I.G. Kevrekidis, S. Sundaresan, Fully developed traveling wave solutions and bubble formation in fluidized beds, J. Fluid Mech. 334 (1997) 157-188

[32] T. Kawaguchi, T. Tanaka, Y. Tsuji, Numerical simulation of twodimensional fluidized beds using discrete element method (comparison between the two- and three models), Powder Technol. 96 (1998) $129-138$

[33] D. Gera, M. Gautam, Y. Tsuji, T. Kawaguchi, T. Tanaka, Computer simulation of bubbles in large-particle fluidized beds, Powder Technol. 98 (1998) 38-47.

[34] J. Ouyang, J.H. Li, Particle-motion-resolved discrete model for simulating gas-solid fluidization, Chem. Eng. Sci. 54 (1999) 2077-2083.

[35] S. Ergun, Fluid flow through packed columns, Chem. Eng. Prog. 48 (1952) 89-94.

[36] C.Y. Wen, Y.H. Yu, Mechanics of fluidization, Chem. Eng. Prog. Symp. Ser. 62 (1966) 100-105.

[37] D. Drew, L. Cheng, R.T. Lahey Jr., The analysis of virtual mass effects in two-phase flow, Int. J. Multiph. Flow 5 (1979) $233-242$

[38] P.D. Minev, U. Lange, K. Nandakumar, A comparative study of twophase flow models relevant to bubble column dynamics, J. Fluid Mech. 394 (1999) 73-96.

[39] G.K. Batchelor, A new theory of the instability of a uniform fluidized bed, J. Fluid Mech. 193 (1988) 75-110.

[40] G.M. Homsy, M.M. Rl-Kaissy, A. Didwania, Instability waves and the origin of bubbles in fluidized beds: Part II. Comparison with theory, Int. J. Multiph. Flow 6 (1980) $305-318$.

[41] S.E. Harris, D.G. Crighton, Solitons, solitary waves, and voidage disturbances in gas-fluidized beds, J. Fluid Mech. 266 (1994) $243-276$.

[42] R. Zenit, M.L. Hunt, C.E. Brennen, Collisional particle pressure measurements in solid-liquid flows, J. Fluid Mech. 353 (1997) $261-283$

[43] J.F. Richardson, W.N. Zaki, Sedimentation and fluidization: part 1, Trans. Inst. Chem. Eng. 32 (1954) 35-53.

[44] R.H. Wilhelm, M. Kwauk, Fluidization of solid particles, Chem. Eng. Prog. 44 (1948) 201-218.

[45] T.-W. Pan, D.D. Joseph, R. Bai, R. Clowinski, V. Sarin, Fluidization of 1204 spheres: simulation and experiment, J. Fluid Mech. 451 (2002) $169-191$. 
[46] D.Z. Zhang, W.B. VanderHeyden, High-resolution three-dimensional numerical simulation of a circulating fluidized bed, Powder Technol. 116 (2001) $133-141$.

[47] D. Gidaspow, Modeling of two-phase flow, Proceeding of 5th International Heat Transfer Conference, JSME-SCEJ, Tokyo, Japan, 1974, pp. $163-168$.

[48] R.W. Lyczkowski, D. Gidaspow, C.W. Solbrig, E.D. Hughes, Characteristics and stability analyses of transient one-dimensional two-phase flow equations and their finite difference approximations, Nucl. Sci. Eng. 66 (1978) 378-396.

[49] G.-S. Jiang, C.-W. Shu, Efficient implementation of weighted ENO schemes, J. Comput. Phys. 126 (1996) 202-228

[50] G.B. Wallis, One-dimensional Two-phase Flow, McGraw-Hill Co., New York, 1969 